\title{
Dehn surgery, rational open books and knot Floer homology
}

\author{
MatTHEW HedDEN \\ Olga Plamenevskaya
}

\begin{abstract}
By recent results of Baker, Etnyre and Van Horn-Morris, a rational open book decomposition defines a compatible contact structure. We show that the Heegaard Floer contact invariant of such a contact structure can be computed in terms of the knot Floer homology of its (rationally null-homologous) binding. We then use this description of contact invariants, together with a formula for the knot Floer homology of the core of a surgery solid torus, to show that certain manifolds obtained by surgeries on bindings of open books carry tight contact structures.
\end{abstract}

57M25, 57M27, 57R17, 57R58

\section{Introduction}

Dehn surgery is the process of excising a neighborhood of an embedded circle (a knot) in a 3-dimensional manifold and subsequently regluing it with a diffeomorphism of the bounding torus. This construction has long played a fundamental role in the study of 3-manifolds, and provides a complete method of construction. If the 3manifold is equipped with extra structure, one can hope to adapt the surgery procedure to incorporate this structure. This idea has been fruitfully employed in a variety of situations.

Our present interest lies in the realm of 3-dimensional contact geometry. Here, contact surgery along Legendrian knots has been an invaluable tool for the study of 3-manifolds equipped with a contact structure. For contact surgery, we start with a Legendrian knot (a knot which is tangent to the contact structure), and perform Dehn surgery in such a way that the contact structure on the knot complement is extended over the surgery solid torus. To guarantee that the extension is unique a condition on the surgery slope is required, and a sufficient condition is that the slope is of the form $1 / k$ with respect to the Legendrian framing; see Ding and Geiges [5] and Kanda [28]. The case when $k=-1$ is perhaps the most well-known, and is often called Legendrian surgery. 
A central goal of this article is to study a different situation in which Dehn surgery uniquely produces a contact manifold. For this we employ an important tool in 3dimensional contact geometry: open book decompositions. An open book decomposition of a 3-manifold $Y$ is equivalent to a choice of fibered knot $K \subset Y$, by which we mean a knot whose complement fibers over the circle so that the boundary of any fiber is a longitude. We refer to $K$ as the binding of the open book. From an open book decomposition, one can produce a contact structure, which is unique, up to isotopy. Note that for this contact structure, the knot $K$ will be transverse to the contact planes. Surgeries on transverse knots were studied in Gay [12], but our perspective is different from [12].

Given a knot $K \subset Y$, denote the manifold obtained by Dehn surgery with slope $p / q$ by $Y_{p / q}$. There is a canonical knot induced by the surgery; namely, the core of the solid torus used in the construction. We denote this knot by $K_{p / q}$. If we perform surgery on a fibered knot $K \subset Y$ then the complement of the induced knot $K_{p / q} \subset Y_{p / q}$ fibers over the circle; indeed, it is homeomorphic to the complement of $K$. However, $K_{p / q}$ is often not fibered in the traditional sense, as the boundaries of the fibers are not longitudes. In fact $K_{p / q}$ will be homologically essential if $p \neq 1$, and so will not have a Seifert surface at all. If $p \neq 0$, then $K$ will be rationally null-homologous, meaning that a multiple of its homology class is zero. We refer to a rationally null-homologous knot whose complement fibers over the circle as a rationally fibered knot, and the corresponding decomposition of the 3-manifold as a rational open book decomposition. Baker, Etnyre and Van Horn-Morris [1] recently showed that a rational open book gives rise to a contact structure, which is unique, up to isotopy. Thus a fibered knot $K \subset Y$ induces a unique contact structure $\xi$ on $Y$, and Dehn surgery on $K$ gives rise to a rationally fibered knot $K_{p / q} \subset Y_{p / q}$ inducing a unique contact structure $\xi_{p / q}$ on $Y_{p / q}$. The purpose of this article is to investigate the relationship between these contact structures.

Our investigation will rely on Heegaard Floer homology, which provides a powerful invariant of contact structures. Denoted $c(\xi)$, this invariant lives in $\widehat{H F}(-Y)$, the Heegaard Floer homology of the manifold $Y$ with its orientation reversed $(\mathbb{F}=\mathbb{Z} / 2 \mathbb{Z}$ coefficients are used throughout, to avoid any sign ambiguities). We study $\xi_{p / q}$ by way of its contact invariant, so it will be useful to understand how to compute the contact invariant associated to a rational open book. Our first theorem states that, as in the null-homologous case, the contact invariant is a function of the knot Floer homology of the binding.

To understand the statement, recall that a rationally null-homologous knot $K \subset Y$ induces a $\mathbb{Z}$-filtration of $\widehat{C F}(-Y)$, that is, a sequence of subcomplexes with integer 
indices:

$$
0 \subset \mathcal{F} \text { (bottom) } \subset \mathcal{F} \text { (bottom }+1) \subset \cdots \subset \widehat{C F}(-Y) .
$$

(See Section 2 for more details on the filtration.) We have:

Theorem 1 Let $K \subset Y$ be a rationally fibered knot, and $\xi_{K}$ the contact structure induced by the associated rational open book decomposition. Then $H_{*}(\mathcal{F}($ bottom $)) \cong$ $\mathbb{F} \cdot\langle c\rangle$. Moreover, if

$$
\iota: \mathcal{F}(\text { bottom }) \rightarrow \widehat{C F}(-Y)
$$

is the inclusion map of the lowest non-trivial subcomplex, then $\iota_{*}(c)=c\left(\xi_{K}\right) \in$ $\widehat{H F}(-Y)$.

In the case that $K$ is fibered in the traditional sense (so that it induces an honest open book decomposition of $Y$ ), this agrees with Ozsváth and Szabó's definition of $c(\xi)$. We also remark that the definition of the filtration depends on a choice of relative homology class, and the class used in the theorem comes from the fiber.

The proof of Theorem 1 uses a cabling argument. More precisely, an appropriate cable of $K$ is a fibered knot in the traditional sense, and results of Baker, Etnyre and Van Horn-Morris [1] relate the contact structure of the resulting open book to that of the original rational open book. We prove the theorem by developing a corresponding understanding of the behavior of the knot filtration under cabling. This is aided by techniques developed in Hedden [18]. We should point out that while the cabling argument shows that $H_{*}(\mathcal{F}$ (bottom) $) \cong \mathbb{F}$, we give an alternate proof of this fact by constructing an explicit Heegaard diagram adapted to a rational open book where the subcomplex in question is generated by a single element, Proposition 3.4. This is a rational analogue of the Heegaard diagram for fibered knots constructed in Ozsváth and Szabó [39], and may be useful for understanding the interaction between properties of the monodromy of a rational open book and those of the contact invariant. By combining the theorem with results of $\mathrm{Ni}$ [32] and Hedden [21] (see also Rasmussen [43]), we arrive at the following corollary.

Corollary 2 Suppose $K \subset L(p, q)$ is a knot in a lens space, and that integral surgery on $K$ yields the 3 -sphere. Then $K$ is rationally fibered, and the associated rational open book induces a contact structure, $\xi_{K}$, with $c\left(\xi_{K}\right) \neq 0$. Regarding $K$ in $-L(p, q)$, the lens space with orientation reversed, we obtain a contact structure $\xi_{\bar{K}}$ also satisfying $c\left(\xi_{\bar{K}}\right) \neq 0$.

Remark [39, Theorem 1.4] shows that non-vanishing contact invariant implies tightness, so the contact structures of the corollary are tight. The corollary also applies to 
knots in L-spaces that admit homology sphere L-space surgeries. The proof of the corollary, contained in Section 3.2, is based on the fact that the Floer homology of knots on which one can perform surgery to pass between L-spaces (manifolds with the simplest Heegaard Floer homology) is severely constrained.

We find this corollary particularly intriguing, due not to the existence of a tight contact structure on $L(p, q)$ induced by $K$, but the additional tight contact structure on $-L(p, q)$. To put this in perspective, if a null-homologous fibered knot $K \subset Y$ induces a tight contact structure on both $Y$ and $-Y$, then the monodromy of the associated open book is isotopic to the identity (otherwise it could not be right-veering with both orientations; see Honda, Kazez and Matić [24]). If one could show that, similarly, there are but a finite number of rationally fibered knots that induce tight contact structures on both $L(p, q)$ and $-L(p, q)$, this would lead to significant progress on the Berge Conjecture, as it would imply that the number of knots in the 3-sphere giving rise to any fixed lens space by Dehn surgery is finite (a fact that would follow from the Berge Conjecture). In any event, we hope that the geometric information provided by the contact structures induced by $K \subset L(p, q)$ can be of aid in the understanding of lens space surgeries.

In another direction, we can use a surgery formula for knot Floer homology to understand the contact invariant of rational open books induced by Dehn surgery. (Here, the 3 manifolds involved do not have to be L-spaces.) Our second main theorem is a non-vanishing result for the contact invariant in this situation.

Theorem 3 Let $K \subset Y$ be a fibered knot with genus $g$ fiber, and $\xi$ the contact structure induced by the associated open book. Let $K_{p / q} \subset Y_{p / q}$ be the rationally fibered knot arising as the core of the solid torus used to construct $p / q$ surgery on $K$, and $\xi_{p / q}$ the contact structure induced by the associated rational open book. Suppose $c(\xi) \in$ $\widehat{H F}(-Y)$ is non-zero. Then $c\left(\xi_{p / q}\right) \in \widehat{H F}\left(-Y_{p / q}\right)$ is non-zero for all $p / q \geq 2 g$.

Note that surgeries with sufficiently negative framings can be realized as Legendrian surgeries. If $(Y, \xi)$ has non-trivial contact invariant, so will any contact structure obtained by Legendrian surgery, regardless of fibering. For this reason, producing tight contact structures on positive Dehn surgeries is typically more challenging, and explains our focus on the realm of positive slope. We should point out, however, that our results have analogues for negative slopes, which can be used to produce contact structures with non-trivial invariants, even in situations where the slope is larger than the maximal Thurston-Bennequin invariant.

Theorem 3 allows us to construct a number of interesting tight contact structures. First, notice that surgeries on the binding of an open book with trivial monodromy 
produce rational open book decompositions for circle bundles over surfaces. Tight contact structures on circle bundles are completely classified (Honda [23] and Giroux [15]), but it is interesting to point out that an existence result follows immediately from Theorem 3: a circle bundle of Euler number $n \geq 2 g$ over a surface of genus $g>0$ carries a tight contact structure with non-zero contact invariant. To list some further families of contact manifolds whose tightness follows from Theorem 3, we turn to the supply of tight contact structures compatible with the genus one open books given in Baldwin [2; 3]. Indeed, tight contact structures supported by open books $(T, \phi)$ (where $T$ is a punctured torus) are completely classified (Baldwin [2], and Honda, Kazez and Matić [25]) in terms of their monodromy. All of these tight contact structures have non-vanishing contact invariants, so Theorem 3 produces, for any $p / q \geq 2$, tight contact structures on manifolds obtained by $p / q$-surgery on the bindings of corresponding open books. Many of these manifolds are L-spaces [3] and thus carry no taut foliations (Ozsváth and Szabó [35, Theorem 1.4]); the family of tight contact manifolds we obtain generalizes a result of Etgü [9]. (Note that an expanded version of [9] extends the results to a wider class of open books than the original arXiv version.) More generally, any L-space obtained by surgery on a fibered knot in an L-space provides an example of a manifold with no taut foliations but a tight contact structure by our theorem. This includes many hyperbolic 3-manifolds. Of course our theorem also shows that performing surgery with slopes in $[2 g, \infty)$ on the binding of any pseudo-Anosov open book decomposition with non-zero contact invariant produces infinitely many hyperbolic manifolds carrying tight contact structures, but presumably many of these manifolds also carry taut foliations.

Our results should also be contrasted to those of Lisca and Stipsicz [31]. They prove that for a knot $K \subset S^{3}$ whose maximal self-linking number equals $2 g(K)-1$, the surgered manifold $S_{r}^{3}(K)$ carries a tight contact structure for all $r \geq 2 g(K)$. While our theorem only applies to fibered knots, it can be used in arbitrary 3 -manifolds. In particular, combining Theorem 3 with Hedden [20, Theorem 5] produces:

Corollary 4 Let $K \subset Y$ be a fibered knot with fiber $F$, and $\xi$ a contact structure on $Y$ with $c(\xi)$ non-zero. Assume that $K$ has a transverse representative in $\xi$ satisfying

$$
s l_{F}(K)=2 g(F)-1 .
$$

Then $K_{p / q} \subset Y_{p / q}$ induces a contact structure $\xi_{p / q}$ with $c\left(\xi_{p / q}\right)$ non-zero, for $p / q \geq 2 g(F)$.

Our result overlaps with [31] for fibered knots in $S^{3}$ with $s l(K)=2 g(K)-1$, but [31] guarantees only the existence of a tight contact structure whereas our result describes a specific supporting open book. In the present context the self-linking condition 
guarantees that the contact invariant of the contact structure induced by $K$ is equal to $c(\xi)$, which, by assumption, is non-zero. This fact follows from a kind of relative adjunction inequality established in [20]. In fact, the contact structure induced by $K$ is the same as $\xi$ : by Etnyre and Van Horn-Morris [11, Theorem 1.1], $\xi$ can only differ from $\xi_{K}$ by extra Giroux torsion, but by Ghiggini, Honda and Van Horn-Morris [13] the presence of Giroux torsion would imply $c(\xi)=0$, so we must have $\xi_{K}=\xi$.

Our proof of Theorem 3 consists of several parts. The first is based on a detailed examination of the knot Floer homology of the induced knot $K_{n} \subset Y_{n}$ for sufficiently large integral surgeries, $n \in \mathbb{Z}$. Building on work of Hedden [19], and Ozsváth and Szabó [36], we give a complete description of the knot Floer homology filtration induced by $K_{n} \subset Y_{n}$ in terms of the filtration induced by $K \subset Y$. Coupled with the description of the contact invariant given by Theorem 1, this proves the theorem for $n \gg 0$. We then obtain the theorem for all integers $n \geq 2 g$ by using an exact sequence for knot Floer homology together with an adjunction inequality. It is worth pointing out that the restriction $n \geq 2 g$ is, in general, sharp (this can be seen from the $(2, k)$ torus knot; Etnyre and Honda [10], and Lisca and Stipsicz [30]). Finally, the theorem is proved for rational slopes $p / q \geq 2 g$ by showing that $\xi_{p / q}$ is obtained from $\xi_{n}$ by Legendrian surgery.

Outline The paper is organized as follows. In Section 2, we discuss the Alexander grading in knot Floer homology, paying particular attention to the case of rationally null-homologous knots. In particular, we discuss how to compute this grading with the help of so-called relative periodic domains.

Section 3 is devoted the proof of Theorem 1. The proof relies on studying the relationship between the knot Floer homology of the binding of an open book and that of its cables. In this section we also produce an explicit Heegaard diagram for a rationally fibered knot with a unique generator for the lowest non-trivial filtered subcomplex in the knot filtration.

In Section 4 we prove Theorem 3. This section includes a detailed discussion of the relationship between the knot Floer homology of $K \subset Y$ and the Floer homology of the induced knot $K_{p / q} \subset Y_{p / q}$.

\section{Acknowledgements}

We are grateful to Jeremy Van Horn-Morris for many helpful conversations, to András Stipsicz and Paolo Lisca for their interest, and to John Etnyre for help with Lemma 4.7. Much of this work was completed at the Mathematical Sciences Research Institute, during the program "Homology Theories for Knots and Links", and at the Banff 
International Research Station during the workshop "Interactions between contact symplectic topology and gauge theory in dimensions 3 and 4" in March 2011. We are very grateful for the wonderful environment provided by both institutions. We also thank the referee for an extremely thorough report. The many thoughtful comments therein increased the quality of the published version of this article in a significant way. Matthew Hedden was partially supported by NSF grant DMS-0906258, NSF CAREER grant DMS-1150872, and an Alfred P Sloan Research Fellowship. Olga Plamenevskaya was partially supported by NSF grant DMS-0805836.

\section{Rationally null-homologous knots and the Alexander grading}

Let $K \subset Y$ be knot. We say that $K$ is rationally null-homologous if $[K]=0 \in H_{1}(Y ; \mathbb{Q})$. This implies that for some positive integer $p$, we have $p \cdot[K]=0$ in $H_{1}(Y ; \mathbb{Z})$, and that there exists a smooth, properly embedded surface $F \subset Y \backslash \nu K$ such that $[\partial F]=p \cdot[K]$. If $p$ is minimal, we call it the order of $K$, and refer to the aforementioned surface as a rational Seifert surface for $K$. Finally, we say that a rationally null-homologous knot is rationally fibered if $Y \backslash v K$ fibers over the circle with fiber a rational Seifert surface. In this section we discuss Alexander gradings in knot Floer homology, with an emphasis on the case of rationally null-homologous knots. For such knots, an Alexander grading can be defined with the help of the relative homology class coming from a rational Seifert surface. This Alexander grading can, in turn, be computed from a so-called relative periodic domain that represents the homology class of the Seifert surface.

Suppose that $K$ is a rationally null-homologous knot in $Y$, represented by a doubly pointed Heegaard diagram $(\Sigma, \boldsymbol{\alpha}, \boldsymbol{\beta}, w, z)$. The knot induces a filtration of the chain complex $\widehat{C F}(Y)$ by the partially ordered set of relative $\operatorname{Spin}^{c}$ structures $\operatorname{Spin}^{c}(Y, K)$ on the knot complement (Ozsváth and Szabó [42, Section 2]). The partial ordering comes from the fact that $\operatorname{Spin}^{c}(Y, K)$ is an $H^{2}(Y \backslash v K, \partial(Y \backslash v K))$-torsor, and this latter group can be endowed with a partial order (note that there is no canonical partial ordering on torsion cyclic summands in $H^{2}(Y \backslash v K, \partial(Y \backslash v K))$, so we simply pick one). The partial ordering restricts to a total ordering on the fibers of the natural filling map [42, Section 2.2]:

$$
G_{Y, K}: \underline{\operatorname{Spin}^{c}}(Y, K) \longrightarrow \operatorname{Spin}^{c}(Y),
$$

where $G_{Y, K}^{-1}(\mathfrak{s})$ consists of relative $\operatorname{Spin}^{c}$ structures that differ by a multiple of the Poincaré dual to the meridian $\operatorname{PD}([\mu])$. 
A relative homology class $\alpha \in H_{2}(Y \backslash v K, \partial(Y \backslash v K))$ allows us to collapse the partial order on $\underline{\operatorname{Spin}^{c}}$ to a total order. Define $\mathrm{A}_{\alpha}: \underline{\operatorname{Spin}^{c}}(Y, K) \rightarrow \mathbb{Z}$ by

$$
\mathrm{A}_{\alpha}(\underline{\mathfrak{s}})=\frac{1}{2}\left\langle c_{1}(\underline{\mathfrak{s}})-\mathrm{PD}([\mu]), \alpha\right\rangle,
$$

where $c_{1}(\underline{\mathfrak{s}}) \in H^{2}(Y \backslash v K, \partial(Y \backslash v K)$ is the relative Chern class of the orthogonal 2-plane field to the relative $\operatorname{Spin}^{c}$ structure, relative to a specific trivialization on the boundary (Ozsváth and Szabó[41, page 627]). This function gives $\underline{\operatorname{Spin}^{c}}$ a total order, and hence a total order on the set of generators for $\widehat{C F}(Y)$, by the function

$$
\mathfrak{s}_{z, w}(-): \mathbb{T}_{\alpha} \cap \mathbb{T}_{\beta} \rightarrow \underline{\operatorname{Spin}^{c}}(Y, K) .
$$

For the purposes of knot Floer homology, the relevant $\alpha \in H_{2}(Y \backslash v K, \partial(Y \backslash v K))$ is the class of a rational Seifert surface, $[F, \partial F] \in H_{2}(Y \backslash v K, \partial(Y \backslash v K))$. In this case, we refer to the function

$$
\mathrm{A}_{[F, \partial F]}(\mathbf{x})=\frac{1}{2}\left(\left\langle c_{1}\left(\mathfrak{s}_{z, w}(\mathbf{x})\right),[F, \partial F]\right\rangle-[\mu] \cdot[F, \partial F]\right)
$$

as the Alexander grading. This depends on the choice of rational Seifert surface, but only through its relative homology class. We will often drop this choice from the notation, letting $\mathrm{A}(\mathbf{x})$ denote the Alexander grading of a generator, defined with respect to an implicit choice of rational Seifert surface (when $b_{1}(Y)=0$ this choice is canonical). The Alexander grading gives rise to a filtration $\mathcal{F}$ on $\widehat{C F}(Y)$ in the standard way, ie, we let

$$
\mathcal{F}(s)=\bigoplus_{\left\{\mathbf{x} \in \mathbb{T}_{\alpha} \cap \mathbb{T}_{\beta} \mid \mathrm{A}(\mathbf{x}) \leq s\right\}} \mathbb{F}\langle\mathbf{x}\rangle
$$

denote the subgroup of $\widehat{C F}(Y)$ generated by intersection points with Alexander grading less than or equal to $s \in \mathbb{Z}$. Positivity of intersections of $J$-holomorphic Whitney disks with the hypersurfaces determined by $z$ and $w$ ensures that $\mathcal{F}(s)$ is a subcomplex, that is, $\partial \mathcal{F}(s) \subset \mathcal{F}(s)$ and hence $\mathcal{F}$ indeed defines a filtration. The associated graded groups are the knot Floer homology groups:

$$
\widehat{H F K}_{*}(Y,[F], K, i):=H_{*}\left(\frac{\mathcal{F}(i)}{\mathcal{F}(i-1)}\right)
$$

The Alexander grading is slightly easier to study if $Y$ is a rational homology sphere (Ni [33]). In this case, if $(Y, K)$ is represented by a doubly pointed Heegaard diagram $(\Sigma, \boldsymbol{\alpha}, \boldsymbol{\beta}, w, z)$, and $\mathbf{x}, \mathbf{y}$ are two generators of $\widehat{C F}(Y)$, consider a curve $a$ in $\mathbb{T}_{\alpha} \subset$ $\operatorname{Sym}^{g}(\Sigma)$ connecting $\mathbf{x}$ to $\mathbf{y}$, and a curve $b$ in $\mathbb{T}_{\beta} \subset \operatorname{Sym}^{g}(\Sigma)$ connecting $\mathbf{y}$ to $\mathbf{x}$. The union $a \cup b$ is a closed curve in $\operatorname{Sym}^{g}(\Sigma)$. Since $b_{1}(Y)=0$, a multiple $k(a \cup b)$ 
bounds a Whitney disk $\phi$, and the filtration difference can be computed by means of this Whitney disk. Indeed,

$$
\mathfrak{s}_{w, z}(\mathbf{x})-\mathfrak{s}_{w, z}(\mathbf{y})=\frac{1}{k}\left(n_{z}(\phi)-n_{w}(\phi)\right) \operatorname{PD}([\mu]),
$$

and this quantity is independent of $\phi$ [33, Lemma 4.2] (see also [41, Lemma 3.11]).

If $b_{1}(Y)>0$, some generators of $\widehat{C F}(Y)$ may not be related by a Whitney disk, although the above formula still holds for $\mathbf{x}, \mathbf{y}$ such that their relative $\operatorname{Spin}^{c}$-structures differ by a multiple of $\operatorname{PD}([\mu])$; this is always the case if $\mathfrak{s}_{z, w}(\mathbf{x})$ and $\mathfrak{s}_{z, w}(\mathbf{y})$ are in the same fiber of the filling map (1). To understand the Alexander grading in the absence of rational Whitney disks we will use "relative periodic domains" to evaluate the grading difference between two generators.

Let $K \subset Y$ be a knot, and let $(\Sigma, \boldsymbol{\alpha}, \boldsymbol{\beta}, z, w)$ be a Heegaard diagram for $(Y, K)$. Connect $z$ to $w$ by an arc $l_{1}$ in $\Sigma$ disjoint from the $\alpha$-curves, and $w$ to $z$ by an arc $l_{2}$ in $\Sigma$ disjoint from the $\beta$-curves. The union $\lambda=l_{1} \cup l_{2}$, when pushed into the respective handlebodies, is a longitude for $K$. We will always consider Heegaard diagrams where such a longitude is fixed for the given knot.

Definition 2.1 Let $K \subset Y$ be a rationally null-homologous knot, and let

$$
(\Sigma, \boldsymbol{\alpha}, \boldsymbol{\beta}, z, w)
$$

be a Heegaard diagram for $(Y, K)$ with a longitude $\lambda$, as above. Let $\mathcal{D}_{1}, \ldots, \mathcal{D}_{r}$ denote the closures of the components of $\Sigma \backslash(\boldsymbol{\alpha} \cup \boldsymbol{\beta} \cup \lambda)$. A relative periodic domain is a 2 -chain $\mathcal{P}=\sum a_{i} D_{i}$, whose boundary satisfies

$$
\partial \mathcal{P}=l \lambda+\sum_{i} n_{i} \alpha_{i}+\sum_{i} m_{i} \beta_{i}
$$

for $l, n_{i}, m_{i} \in \mathbb{Z}$.

Remark 2.2 Our definition is a generalization of the notion of periodic domain (Ozsváth and Szabó [38, Definition 2.14]). A periodic domain is a two chain as above, satisfying $l=0$ and $n_{w}(\mathcal{P})=0$. We drop this latter condition since our purpose for relative periodic domains is to study the relative Alexander grading. This quantity depends only on the relative multiplicities of $\mathcal{P}$, and is not affected by the addition of the homology class $[\Sigma]$.

A relative periodic domain $\mathcal{P}$ naturally gives rise to a relative homology class $[\mathcal{P}] \in$ $H_{2}(Y \backslash v K, \partial(Y \backslash v K))$, in the same way that periodic domains give rise to homology classes in $H_{2}(Y)$. Indeed, a relative periodic domain is a 2-chain whose boundary 
consists of a union of copies of $\lambda$ and complete $\alpha$ - and $\beta$-curves. Capping off the $\alpha$ - and $\beta$-curves with the disks that they bound in their respective handlebodies, we arrive at a 2 -chain whose boundary lies on $\lambda$ or, up to homotopy, on $\partial(Y \backslash v K)$. In other words, we obtain a cycle in the relative chain group $C_{2}(Y \backslash v K, \partial(Y \backslash v K))$. We denote the corresponding homology class by $[\mathcal{P}]$. In fact, the correspondence is reversible; that is, any relative homology class comes about by capping off a relative periodic domain. Since we have no need for this fact we leave the details (a standard Mayer-Vietoris argument) to the reader.

The Alexander grading is defined in terms of the relative homology class of a rational Seifert surface. Thus our primary interest lies in those relative periodic domains whose homology class agrees with some specific rational Seifert surface $F$. To this end, observe that if $K$ has order $p$, then $\partial F$ will wrap $p$ times around $K$. Thus for a relative periodic domain $\mathcal{P}$ whose homology class agrees with $F$, the longitude $\lambda$ will appear with multiplicity $p$ in $\partial \mathcal{P}$.

The following lemma shows that the relative Alexander grading difference between generators $\mathbf{x}, \mathbf{y}$ is determined by the multiplicities of $\mathcal{P}$.

Lemma 2.3 Let $K \subset Y$ be a rationally null-homologous knot and $\mathcal{P}$ be a relative periodic domain whose homology class equals that of a fixed rational Seifert surface $F$. Let $\mathbf{x}, \mathbf{y} \in \mathbb{T}_{\alpha} \cap \mathbb{T}_{\beta}$. Then

$$
\mathrm{A}(\mathbf{x})-\mathrm{A}(\mathbf{y})=n_{\mathbf{x}}(\mathcal{P})-n_{\mathbf{y}}(\mathcal{P})
$$

where $\mathrm{A}$ is the Alexander grading with respect to $F$, defined by (3).

Proof Recalling the definition of $\mathrm{A}$, we need to evaluate the quantity

$$
\frac{1}{2}\left(\left\langle c_{1}\left(\mathfrak{s}_{w, z}(x)\right),[F, \partial F]\right\rangle-\left\langle c_{1}\left(\mathfrak{s}_{w, z}(y)\right),[F, \partial F]\right\rangle\right)=\left\langle\mathfrak{s}_{w, z}(\mathbf{x})-\mathfrak{s}_{w, z}(\mathbf{y}),[F, \partial F]\right\rangle .
$$

By [41, Lemma 3.11]

$$
\mathfrak{s}_{w, z}(\mathbf{x})-\mathfrak{s}_{w, z}(\mathbf{y})=\operatorname{PD}(\epsilon(\mathbf{x}, \mathbf{y}))=\operatorname{PD}\left(\left[\gamma_{\mathbf{x}}-\gamma_{\mathbf{y}}\right]\right)
$$

where $\gamma_{\mathbf{x}}$ is the union of gradient trajectories connecting index 1 and index 2 critical points of the Morse function that pass through the coordinates $x_{i}$ of $\mathbf{x}=\left(x_{1}, \ldots, x_{g}\right)$, and $\gamma_{\mathbf{y}}$ is a similar union of gradient trajectories passing through the coordinates of $\mathbf{y}$. Therefore, it suffices to calculate the intersection number of the closed curve $\gamma_{\mathbf{x}}-\gamma_{\mathbf{y}}$ with the surface $F$. To this end, recall that the homology class of $[F, \partial F]$ is constructed from the periodic domain $\mathcal{P}$ by capping off any $\alpha$ - and $\beta$-curves appearing in $\partial \mathcal{P}$ (with multiplicity) with the compressing disks bounded by the curve in the corresponding handlebody. 
If $x_{i} \in \mathbf{x}$ (resp. $y_{i} \in \mathbf{y}$ ) lies in the interior of $\mathcal{P} \subset \Sigma$, then the intersection of $F$ with $\gamma_{\mathbf{x}}\left(\right.$ resp. $\left.\gamma_{\mathbf{y}}\right)$ equals the multiplicity $\bar{n}_{\mathbf{x}_{i}}(\mathcal{P})\left(\operatorname{resp} . \bar{n}_{\mathbf{y}_{i}}(\mathcal{P})\right)$. If $x_{i} \in \partial \mathcal{P}$ then it does not contribute to the intersection number, as the surface can be perturbed so that the compressing disk for the corresponding $\alpha$ - or $\beta$-curve is replaced by a normal translate that is disjoint from $\gamma_{\mathbf{x}}$. It remains to observe that contributions from such boundary points cancel in the expression $n_{\mathbf{x}}(\mathcal{P})-n_{\mathbf{y}}(\mathcal{P})$, since every $\alpha$-curve and every $\beta$-curve contains exactly one coordinate of $\mathbf{x}$ and exactly one coordinate of $\mathbf{y}$.

\section{The contact invariant for rational open books}

Let $K \subset Y$ be a rationally fibered knot. Such a knot induces a rational open book decomposition and, subsequently, a contact structure $\xi_{K}$ [1]. The purpose of this section is to understand the Ozsváth-Szabó contact invariant of $\xi_{K}$ in terms of the knot Floer homology of $K$. More precisely, the "bottom" filtered subcomplex in the filtration of $\widehat{C F}(-Y)$ induced by $K$ has homology $\mathbb{F}$, generated by a vector $c$ (this can be seen in many ways, and follows from both Propositions 3.1 and 3.4 below). The main result of this section, Theorem 1 , shows that

$$
c\left(\xi_{K}\right)=\iota_{*}(c)
$$

where $\iota: \mathcal{F}$ (bottom) $\rightarrow \widehat{C F}(-Y)$ is the inclusion map. That is, the contact invariant of $\xi_{K}$ is the image of the generator of the homology of the bottom filtered subcomplex in the Floer homology of $-Y$, under the natural inclusion-induced map. When $K$ is fibered in the traditional sense, this is simply Ozsváth and Szabó's definition [39, Definition 1.2].

We prove Theorem 1 by considering an honest open book that results from an appropriate cabling of $K$. Let $K_{P, P n+1}$ denote the $(P, P n+1)$-cable of $K$. It is clear that $\left[K_{P, P n+1}\right]=P \cdot[K] \in H_{1}(Y ; \mathbb{Z})$. Thus for $P$ equal to the order of $K$, the cables will be null-homologous. Moreover, such cables are fibered in the traditional sense, provided that $K$ is rationally fibered. When $P, n>0$, it follows from [1, Theorem 1.8] that $\xi_{K}$ is isotopic to $\xi_{K_{P, P n+1}}$. The theorem will follow by understanding the relation between the knot Floer complex of a given knot and its cable. This is accomplished by Proposition 3.1, which generalizes the cabling result of [18]. While our results show that $H_{*}(\mathcal{F}$ (bottom $\left.)\right) \cong \mathbb{F}$, we conclude the section by constructing an explicit Heegaard diagram adapted to a rational open book decomposition. For this Heegaard diagram the bottom Floer homology group is represented by a complex with a single generator. With the plan in place, we begin. 


\subsection{The contact invariant and cabling}

In this subsection we prove Theorem 1. The key tool is Proposition 3.1, which establishes a relationship between the Floer complex of a rationally null-homologous knot and that of its sufficiently positive cables.

The result states that the knot Floer homology groups of a knot and its sufficiently positive cables are equal in the "topmost" Alexander gradings. To make this precise, recall that the Alexander grading depends on a choice; namely, the relative homology class of a rational Seifert surface (3). To specify how this choice is made, fix a rational Seifert surface $F$ for the knot $K$. We construct a rational Seifert surface $F^{\prime}$ for the cable $K^{\prime}=K_{P, P n+1}$ as follows. If $K$ has order $p$ in $H_{1}(Y)$, then $F$ intersects $\partial \nu K$ in a curve $s$ that wraps $p$ times around the longitude. The cable $K^{\prime}$ has order $p^{\prime}=p / \operatorname{gcd}(P, p)$. Thus a rational Seifert surface $F^{\prime}$ for $K^{\prime}$ must meet $v K^{\prime}$ in a curve $s^{\prime}$ that is null-homologous in $Y \backslash v K^{\prime}$ and wraps $p^{\prime}$ times around the longitude. We can assume that the neighborhood of the cable is contained inside that of the knot, $v K^{\prime} \subset v K$. To construct $F^{\prime}$, we take $R=P / \operatorname{gcd}(P, p)$ parallel copies of $F$, and glue them to an oriented properly embedded surface in $v K \backslash v K^{\prime}$ whose boundary consists of $s^{\prime}$ and $R$ parallel copies of $s$.

Proposition 3.1 Let $K \subset Y$ be a rationally null-homologous knot, and $K^{\prime}=K_{P, P n+1}$ its $(P, P n+1)$-cable. Fix a rational Seifert surface $F$ for $K$, and consider the corresponding rational Seifert surface $F^{\prime}$ for $K^{\prime}$, described above. Then for all $n \gg 0$ sufficiently large, we have

$$
\widehat{H F K}_{*}(Y,[F], K, \text { top })=\widehat{H F K}_{*}\left(Y,\left[F^{\prime}\right], K^{\prime}, \text { top }^{\prime}\right) .
$$

Remark 3.2 Disregarding gradings, an isomorphism between the groups above can be shown for all $n$ using sutured manifold decompositions [32, Corollary 5.9] (see also Juhász [27]). However, the strategy of our proof will be essential for our understanding of how the contact invariants of a rational open book and its cables are related.

Proof For the case where $K$ is a knot in $S^{3}$, this statement was established in [18] (see also [33] for a generalization to the case where $Y$ is a rational homology sphere). However, the proofs from [18] and [33] use Whitney disks to compare the Alexander gradings of different generators of $\widehat{C F}(Y)$; when $b_{1}(Y)>0$, this proof no longer works since there may be no Whitney disks. Instead, we will use Lemma 2.3 to compare the gradings in the Heegaard diagrams of $[18 ; 33]$.

Let $F$ be a rational Seifert surface for $K$. We can find a doubly pointed Heegaard diagram $(\Sigma, \boldsymbol{\alpha}, \boldsymbol{\beta}, w, z)$ for $(Y, K)$, together with a longitude $\lambda_{0}$ on $\Sigma$ and a relative 
periodic domain $\mathcal{P}$ representing $F$. It will be convenient to enumerate the $\alpha$ - and $\beta$-curves by the index set $\{0,1, \ldots, g-1\}$, and to suppress the indices of $\alpha_{0}=\alpha$ and $\beta_{0}=\beta$. We assume that $\lambda_{0}$ connects points $z$ and $w$, intersects $\beta_{0}$ at a single point, and is disjoint from all other $\beta$-curves, so that $\beta$ represents a meridian for $K$. We also require that $\beta$ intersects $\alpha$ at a single point and is disjoint from the other $\alpha$-curves. The relative periodic domain $\mathcal{P}$ gives rise to the homological relation

$$
p \lambda_{0}+q \beta+\sum_{i=0}^{g-1} r_{i} \alpha_{i}+\sum_{i=1}^{g-1} q_{i} \beta_{i}=0 .
$$

The multiplicities of $\mathcal{P}$ in the components of $\Sigma \backslash\left(\boldsymbol{\alpha} \cup \boldsymbol{\beta} \cup \lambda_{0}\right)$ can be determined as follows: pick a component $\mathcal{D}_{0} \in \Sigma \backslash\left(\boldsymbol{\alpha} \cup \boldsymbol{\beta} \cup \lambda_{0}\right)$ and assign the multiplicity of $\mathcal{P}$ in $\mathcal{D}_{0}$ to be zero. The multiplicity of $\mathcal{P}$ in any other component $\mathcal{D}_{i}$ is the algebraic intersection number $\# \gamma \cap \partial \mathcal{P}$ of an oriented arc from $\mathcal{D}_{i}$ to $\mathcal{D}_{0}$ with the sum of curves in (4). It is customary to fix the multiplicity of the component containing $w$ to be zero; however, in the argument below we find it convenient to fix the multiplicity of another component (recall Definition 2.1 places no restriction on $n_{w}(\mathcal{P})$ ).

We need to construct a Heegaard diagram representing the $(P, P n+1)$-cable of $K$, and to understand the relation between the top filtration level of the homology of $K$ and its cable. To this end, we first replace the longitude $\lambda_{0}$ with an $n$-framed longitude $\lambda=\lambda_{0}+n \beta$. (See Figure 1.) Of course, $\lambda$ is still a longitude for the same knot $K$, but rewriting (4) as

$$
p \lambda+(q-p n) \beta+\sum_{i=0}^{g-1} r_{i} \alpha_{i}+\sum_{i=1}^{g-1} q_{i} \beta_{i}=0,
$$

we get a relation that gives rise to a new periodic domain for $K$. Note that the homology class of this domain equals $[F]$, and its boundary includes $\lambda$.

As the next step, we modify the curve $\beta$ by a finger move. This serves to produce a diagram, which can represent both the knot $K$ and its $(P, P n+1)$-cable, via different placement of marked points. Indeed, we perform a $(P-1)$-fold finger move of $\beta$ along $\lambda$, and replace the basepoint $z$ by $z^{\prime}$ as in Figure 2. The diagram $\left(\Sigma, \boldsymbol{\alpha}, \boldsymbol{\beta}, w, z^{\prime}\right)$ now represents the cable $K^{\prime}$. The diagram also represents the original knot $K$ if we introduce another basepoint $t$, as in Figure 2. The longitude $\lambda$ can be now thought of as a closed curve that passes through $w$ to $t$ so that it intersects $\beta$ once (on the short arc segment joining $w$ and $t$ ) and is disjoint from the other $\beta$ 's. A longitude $\Lambda$ for the cable can be obtained in a similar fashion: connect $w$ and $z^{\prime}$ by a short arc intersecting $\beta P$ times and disjoint from all $\alpha$ 's, then connect $w$ and $z^{\prime}$ by an arc disjoint from all $\beta$ to construct a simple closed curve. 


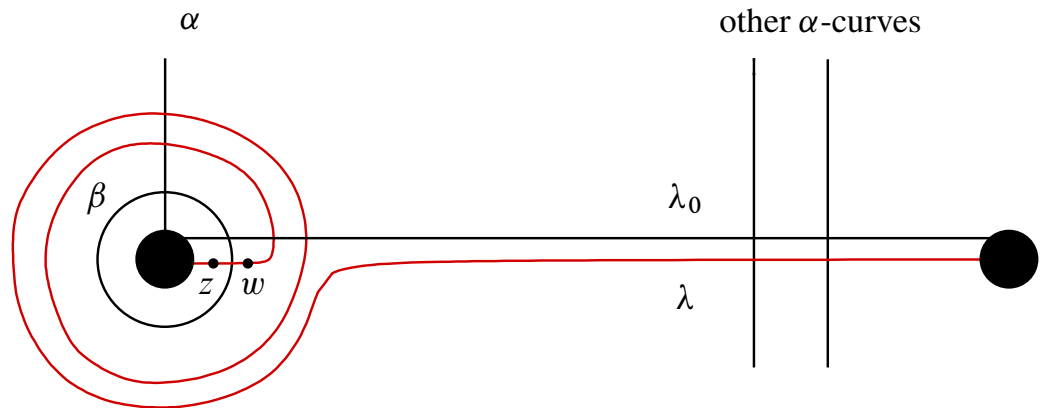

Figure 1: A diagram $(\Sigma, \boldsymbol{\alpha}, \boldsymbol{\beta}, w, z)$ for the knot $K$. The meridian $\beta$, the longitude $\lambda_{0}$, and the $n$-framed longitude $\lambda$ are shown. (Here, $n=2$.) Note that $\lambda_{0}$ is drawn with a shift to make the figure clearer (both $\lambda$ and $\lambda_{0}$ must pass through $w$ and $z$ ).

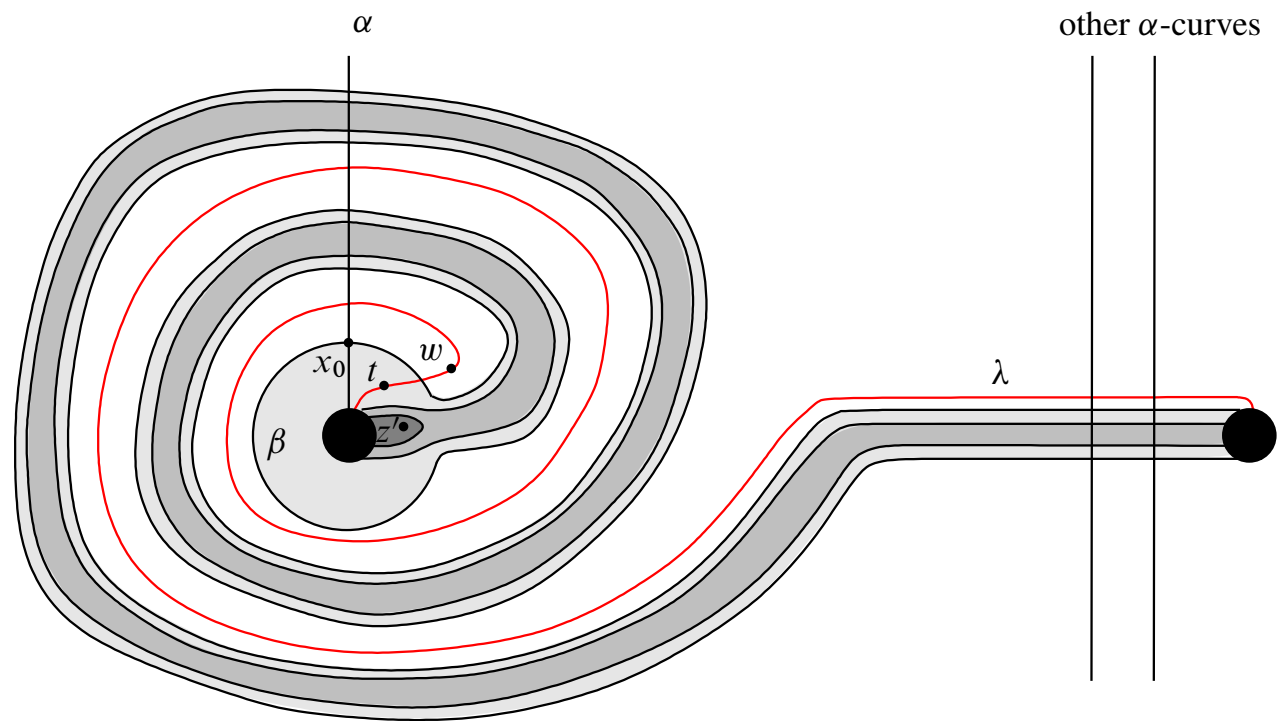

Figure 2: The diagram after a $(P-1)$-fold finger move. In the figure, $P=3, n=2$, so $\left(\Sigma, \boldsymbol{\alpha}, \boldsymbol{\beta}, w, z^{\prime}\right)$ represents a $(3,7)$-cable of the original knot represented by $(\Sigma, \boldsymbol{\alpha}, \boldsymbol{\beta}, w, t)$.

We use the diagram from Figure 2 to locate the generators of the highest filtration level for $K$ and for $K^{\prime}$. We begin with $K$. The relation (5) produces a periodic domain $\mathcal{P}_{n}$ in this diagram; we compute the multiplicities of $\mathcal{P}_{n}$. We are free to pick a multiplicity zero component; we take the component $\mathcal{D}_{0}$ to be outside of the winding region (eg, the top right corner in Figure 2). Then, it is obvious that the multiplicities of $\mathcal{P}_{n}$ are independent of $n$ outside of the winding region. Within the winding region, however, 
the multiplicities increase towards the center of the spiral formed by $\lambda$. The finger move creates a number of parallel copies of $\beta$, and as one moves towards the center of the finger the multiplicities of $\mathcal{P}$ decrease: this is clear if we examine multiplicities of $\mathcal{P}$ in various regions as shown in Figure 3.

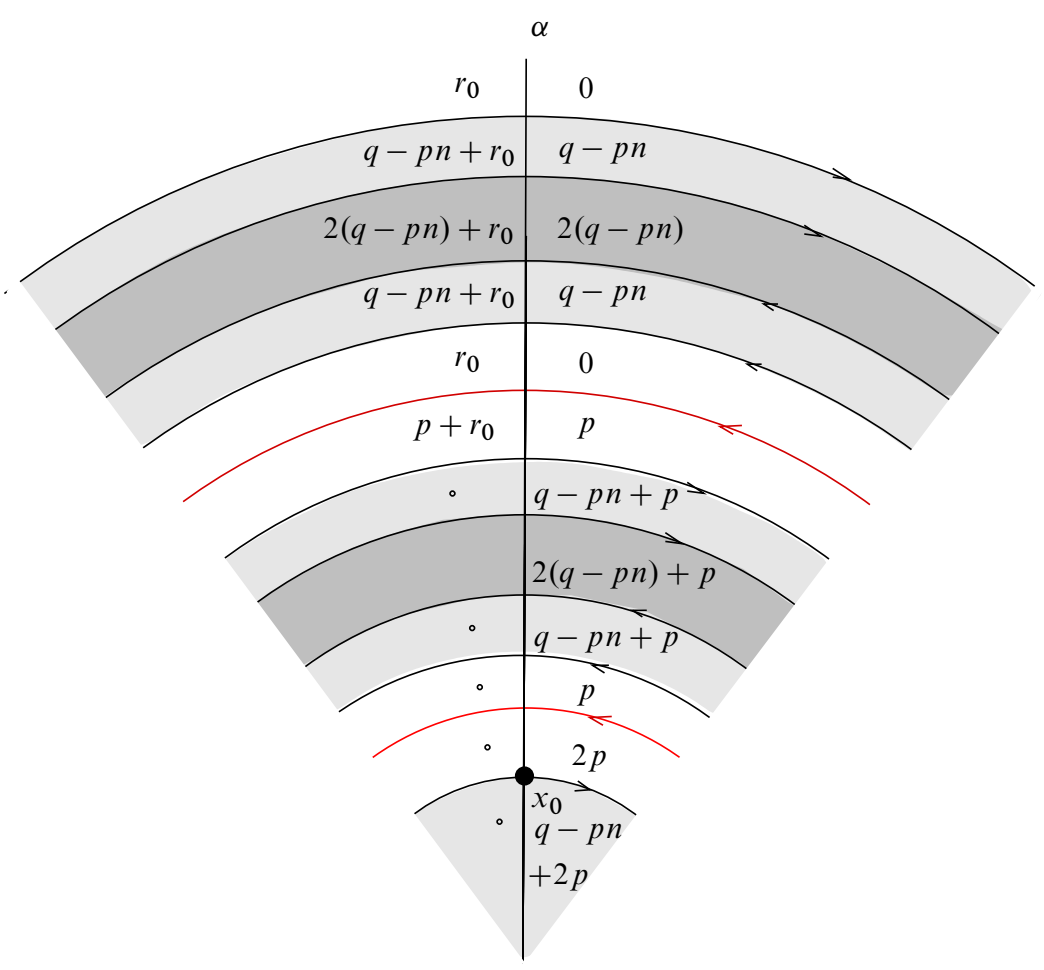

Figure 3: A 2-fold finger and the multiplicities of the periodic domain $\mathcal{P}_{n}$ given by equation (5). (The case $P=3, n=2$ is illustrated.) The meridian $\beta$ is shown in black, the longitude $\lambda$ in red. (The dots in the bottom regions to the left of $\alpha$ replace the multiplicities that are not shown to save space. The multiplicities in these regions differ by $r_{0}$ from the multiplicities in the corresponding regions to the right of $\alpha$.) When $n$ is large, the multiplicities inside the finger are smaller than the multiplicities outside, and the multiplicities increase as we move towards the center of the $\lambda$-spiral in Figure 2.

These considerations show that of the intersection points of $\alpha$ and $\beta$, the point $x_{0}$ (shown in Figure 2) has the highest multiplicity. Since this multiplicity is proportional to $n$, making $n$ large enough ensures that the generators of $\widehat{C F K}(Y, K)$ with the 
highest multiplicities are given by the set $x_{0} \times C$, where $C$ is the set of $(g-1)-$ tuples of intersection points of $\alpha_{1}, \ldots, \alpha_{g-1}$ and $\beta_{1}, \ldots, \beta_{g-1}$ that have the highest multiplicities among all such $(g-1)$-tuples.

To understand the Alexander gradings for the cable, $K^{\prime}$, we must find a relative periodic domain representing $F^{\prime}$ in the same diagram. We now turn our attention to this task.

Consider the cable $K^{\prime}$ and its longitude $\Lambda$. The curve $\Lambda$ is homologous to $P\left(\lambda_{0}+\right.$ $n \beta)+\beta=P \lambda+\beta$. As we already remarked, $\Lambda$ can be drawn in Figure 2 as a union of two arcs connecting $w$ to $z^{\prime}$ : one arc intersects $\beta P$ times and is disjoint from all other $\alpha$ 's and $\beta$ 's; the other arc only intersects the $\alpha$-curves and is disjoint from all $\beta$ 's. We omit the figure illustrating $\Lambda$; the region analogous to the one in Figure 3, with $\Lambda$ replacing $\lambda$, is shown in Figure 4. This figure will be useful for computing multiplicities of the periodic domain that we now describe.

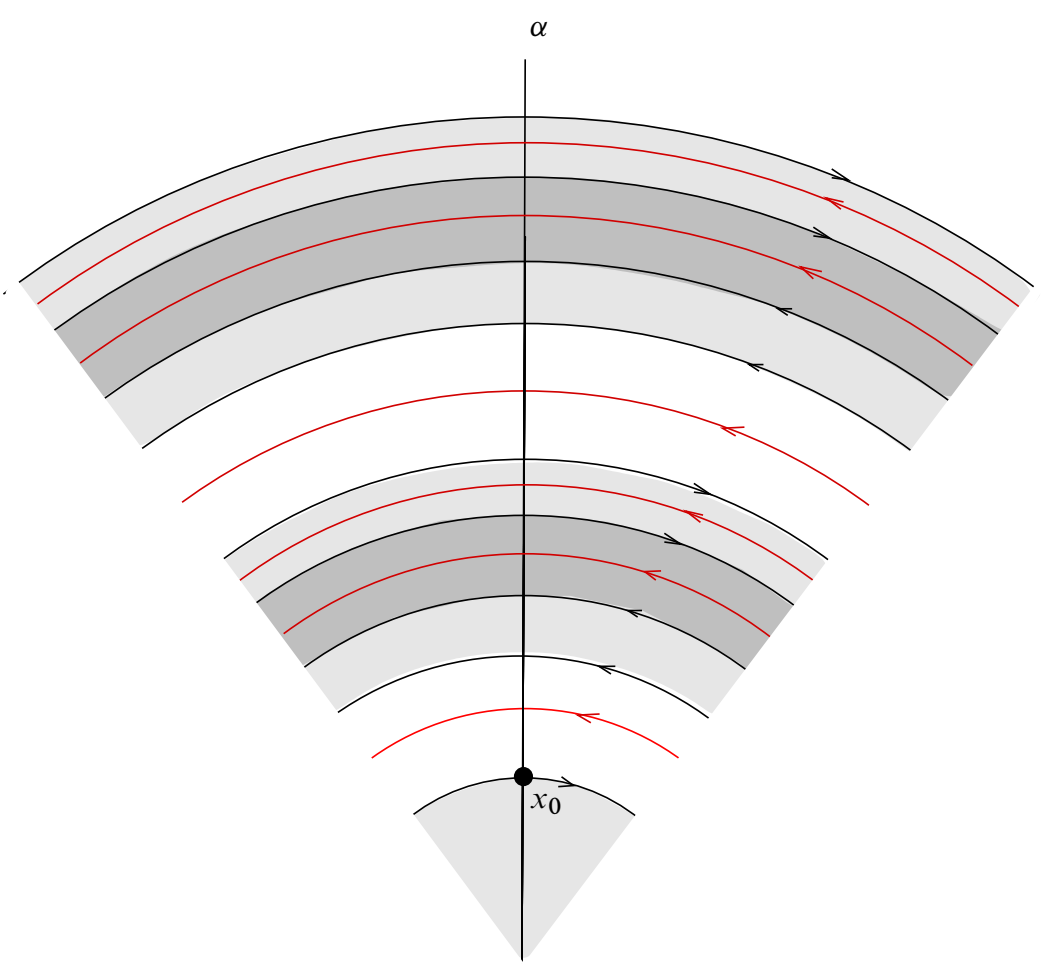

Figure 4: A portion of the Heegaard diagram for the cable for the cable $K^{\prime}$. (A $(3,7)$-cable is illustrated.) The longitude $\Lambda$ is shown in red. 
Multiplying Relation (5) by $R$ and using the fact that $\Lambda$ is homologous to $P \lambda+\beta$ yields the relation

$$
p^{\prime} \Lambda+\left(R q-R p n-p^{\prime}\right) \beta+R\left(\sum_{i=0}^{g-1} r_{i} \alpha_{i}+\sum_{i=1}^{g-1} q_{i} \beta_{i}\right)=0 .
$$

This relation produces a rational periodic domain $\mathcal{P}^{\prime}$ for the cable $K^{\prime}$. It is clear that $\left[\mathcal{P}^{\prime}\right]=\left[F^{\prime}\right] \in H_{2}\left(Y \backslash v K^{\prime}, \partial\left(Y \backslash v K^{\prime}\right)\right)$.

We compute the multiplicities of $\mathcal{P}^{\prime}$ in the winding region. As in Figure 3, we start with 0 in the upper right corner of Figure 4. As we move down along $\alpha$ on the right hand side, the multiplicities, for the case of the $(3,7)$-cable, are

0, $R q-R p n-p^{\prime}, R q-R p n, 2(R q-R p n)-p^{\prime}, 2(R q-R p n), R q-R p n+p^{\prime}, 2 p^{\prime}$, $3 p^{\prime}, R q-R p n+2 p^{\prime}, R q-R p n+3 p^{\prime}, 2(R q-R p n)+2 p^{\prime}, 2(R q-R p n)+3 p^{\prime}$,

$$
R q-R p n+4 p^{\prime}, 5 p^{\prime}
$$

$6 p^{\prime}, R q-R p n+5 p^{\prime}, \ldots$

To the left of $\alpha$, the multiplicities differ by $R r_{0}$ from those in the corresponding regions to the right of $\alpha$. The pattern continues if $n$ is larger, ie, if there is more winding; if $p$ is larger, ie, there are more folds on the finger, there will be additional summands $\left(R q-R p n-p^{\prime}\right)$ as we move towards the center of the finger.

Now we look for generators with highest multiplicities with respect to $\mathcal{P}^{\prime}$. As before, outside of the winding region these multiplicities are independent of $n$. Moreover, we have

$$
\bar{n}_{x}\left(\mathcal{P}^{\prime}\right)=R \bar{n}_{x}(\mathcal{P}) .
$$

For the intersection points with one coordinate on $\beta$, the above relation no longer holds, but the multiplicities of $\mathcal{P}^{\prime}$ behave similarly to the multiplicities of $\mathcal{P}$, increasing towards the center of the winding region and decreasing towards the center of the finger. It follows that when $n$ is large, the top filtration level of $\widehat{C F K}\left(Y, K^{\prime}\right)$ is given by $x_{0} \times C^{\prime}$, where $C^{\prime}$ is defined, analogous to $C$, as the set of $(g-1)$-tuples with the highest multiplicity. Moreover, (6) shows that the set $C$ is identical to the set $C^{\prime}$. This identifies the generators in the top filtration levels of

$$
\widehat{C F K}(Y,[F], K) \text { and } \widehat{C F K}\left(Y,\left[F^{\prime}\right], K^{\prime}\right) .
$$

To identify the homologies in the top grading level, observe that the differentials on $\widehat{C F K}(Y,[F], K$, top $)=\widehat{C F K}\left(Y,\left[F^{\prime}\right], K^{\prime}\right.$, top $\left.{ }^{\prime}\right)$ must both count holomorphic Whitney disks with $n_{w}=n_{t}=n_{z^{\prime}}=0$ (see [18, Proof of Lemma 3.6]); thus, the chain 
complexes $(\widehat{C F K}(Y,[F], K$, top $), \partial)$ and $\left(\widehat{C F K}\left(Y,\left[F^{\prime}\right], K^{\prime}\right.\right.$, top $\left.\left.^{\prime}\right), \partial\right)$ are the same. This completes the proof of the theorem in the case that the Heegaard diagram chosen has the property that there are no generators with Alexander grading bigger than "top", where "top" means "largest number $d$ such that

$$
\widehat{H F K}(Y,[F], K, d)
$$

is non-trivial". Strictly speaking, it could be the case that the largest Alexander grading represented by generators of the original Heegaard diagram (before the finger move) has homology equal to zero. In this case, however, the full proof of [18, Theorem 1.2] could be copied and would produce the desired isomorphism for the topmost non-vanishing Floer homology groups. Indeed, the proof of [18, Theorem 1.2] does not require a Heegaard diagram where the knot Floer complex in the top Alexander grading has non-trivial homology. This is because the theorem calculates the top $M$ groups of the cable in terms of the filtered subcomplexes of the companion knot $K$, where $M$ can be as large as one wishes (at the expense of increasing the cabling parameter $n$ ). For a similar but more concise way to deal with the same issue, see [33, Proposition 5.5]. Alternatively, since we are only interested in the top non-vanishing knot Floer homology group, it is sufficient to find a Heegaard diagram where this group has the highest Alexander grading on the chain level. For the case relevant to this article, that of a rationally fibered knot, we construct such a Heegaard diagram in Proposition 3.4.

\subsection{Proof of Theorem 1}

Let us recall the statement in a slightly more precise form:

Theorem 1 Let $K \subset Y$ be a rationally fibered knot with rational fiber $F$, and let $c\left(\xi_{K}\right)$ denote the contact invariant of the contact structure $\xi_{K}$ induced by the associated rational open book decomposition. Then $H_{*}(\mathcal{F}(-Y,[F], K$, bottom $)) \cong \mathbb{F} \cdot\langle c\rangle$. Moreover, $c\left(\xi_{K}\right)=\iota_{*}(c)$, where

$$
\iota: \mathcal{F}(-Y,[F], K \text {, bottom }) \rightarrow \widehat{C F}(-Y),
$$

is the inclusion map of the subcomplex.

Proof Suppose $K$ has order $p$ in $H_{1}(Y)$. To establish the lemma, we will consider a cable $K^{\prime}=K_{p, p n+1}$, with large $n>0$. Then $K^{\prime}$ is a null-homologous fibered knot inducing an honest open book compatible with $\xi_{K}$ [1, Theorem 1.8]. (The page $F^{\prime}$ of the open book for $K^{\prime}$ can be constructed from $F$ by the procedure described before the statement of Proposition 3.1, provided that we take a Thurston norm minimizing surface in $v K \backslash v K^{\prime}$ as the interpolating surface between $K^{\prime}$ and $\partial F$ ). 
Since $K^{\prime}$ is null-homologous, the results of [39] apply; thus

$$
H_{*}\left(\mathcal{F}\left(-Y,\left[F^{\prime}\right], K^{\prime}, \text { bottom }\right)\right) \cong \mathbb{F}\langle c\rangle
$$

and

$$
c\left(\xi_{K^{\prime}}\right)=\iota_{*}^{\prime}(c),
$$

where $\iota^{\prime}$ is the inclusion map for the cable. Moreover, since positive cabling doesn't change the contact structure, we have

$$
c\left(\xi_{K}\right)=c\left(\xi_{K^{\prime}}\right) .
$$

If we now reverse the orientation of the Heegaard surface in the proof of Proposition 3.1, this has the effect of changing the oriented manifold from $Y$ to $-Y$. It also has the effect of changing the sign of the multiplicities of the rational periodic domains. This reverses the Alexander grading (up to a translation), and proves

$$
\mathcal{F}(-Y,[F], K, \text { bottom })=\mathcal{F}\left(-Y,\left[F^{\prime}\right], K^{\prime} \text {, bottom }\right) .
$$

Let $c$ denote a generator of the homology of this complex. Since the (singly pointed) Heegaard diagram for $-Y$ is independent of the additional basepoint used to specify $K$ or $K^{\prime}$, we have

$$
\iota_{*}(c)=\iota_{*}^{\prime}(c)
$$

Indeed, the respective inclusion maps can be obtained by taking a cycle representative for $c$ and considering the homology class it represents in $\widehat{H F}(-Y)$ by forgetting the respective additional basepoint. Combining (7), (8) and (9) yields the result.

Proof of Corollary 2 Suppose that integer surgery on $K \subset L(p, q)$ is the 3-sphere. Then there is an induced knot $K^{\prime} \subset S^{3}$ on which $\pm p$ surgery produces $L(p, q)$ (the core of the surgery torus). In this situation, [32, Theorem 1.1] implies that $K^{\prime}$ is fibered, and hence $K$ is rationally fibered. By reflecting $K^{\prime}$, if necessary, we may assume the surgery slope is $+p$ (this may change the orientation of $L(p, q)$, but as we ultimately consider both orientations on $L(p, q)$ this point will not affect the argument). Now [21, Theorem 1.4] states that either $p \geq 2 g\left(K^{\prime}\right)$, in which case

$$
\text { rk } \widehat{H F K}(L(p, q), K)=\operatorname{rk} \widehat{H F}(L(p, q))=p,
$$

or $p=2 g\left(K^{\prime}\right)-1$, in which case rk $\widehat{H F K}(L(p, q), K)=\operatorname{rk} \widehat{H F}(L(p, q))+2=p+2$. The latter case, however, is ruled out by [17, Theorem 1.2], and thus the rank of the 
knot Floer homology of $K$ is equal to the rank of the Floer homology of the manifold in which it sits. This immediately implies that the inclusion

$$
\iota: \mathcal{F}(L(p, q), K \text {, bottom }) \rightarrow \widehat{C F}(L(p, q))
$$

is injective on homology: the homology of $\mathcal{F}(L(p, q), K$, bottom) is the bottom knot Floer homology group, which survives under the spectral sequence from the knot Floer homology of $K$ to $\widehat{H F}(L(p, q))$ by the equality of ranks (10). Thus $0 \neq c\left(\xi_{\bar{K}}\right) \in \widehat{H F}(-(-L(p, q)))$. Since reversing the orientation of $L(p, q)$ changes neither the rank of the Floer homology of $K$ nor the ambient manifold, the inclusion

$$
\iota: \mathcal{F}(-L(p, q), K \text {, bottom }) \rightarrow \widehat{C F}(-L(p, q))
$$

is also injective on homology, indicating that the contact structure $\xi_{K}$ induced by $K$ on $L(p, q)$ also has non-vanishing invariant.

Remark 3.3 The corollary is somewhat more general. Indeed, let $K \subset Y$ be a knot in an integer homology sphere L-space whose complement is irreducible, and let $K_{n} \subset Y_{n}$ be the induced knot. Then if $Y_{n}$ is an L-space and $n \geq 2 g(K)$, the conclusion holds, that is, $K_{n}$ is rationally fibered and induces a tight contact structure on both $Y_{n}$ and $-Y_{n}$.

\subsection{A Heegaard diagram for rationally fibered knots}

We can mimic the construction in [39] to pinpoint $c(\xi)$ as the homology class of a specific generator in a particular Heegaard diagram constructed from the open book.

Proposition 3.4 Let $K \subset Y$ be a rationally fibered knot whose rational fiber surface has a single boundary component. There is a Heegaard diagram adapted to $(Y, K)$ so that $\mathcal{F}\left(-Y, K,[F]\right.$, bottom) is generated by a single intersection point $\mathbf{c} \in \mathbb{T}_{\mathbf{f f}} \cap \mathbb{T}_{\mathbf{f i}}$. Thus $c\left(\xi_{K}\right)=[\mathbf{c}] \in \widehat{H F}(-Y)$.

Proof We adapt [39, Theorem 1.1] to construct the required Heegaard diagram. Since $K$ is fibered, the complement of $K$ has a Dehn filling $Y_{0}$, which fibers over $S^{1}$. We first construct a Heegaard diagram for $Y_{0}$, and then recover the desired diagram for $Y$ by a rational surgery.

Let $F$ be the rational Seifert surface for $K$; capping it off, we obtain a closed surface $\widehat{F}$ of genus $g$. We first follow the procedure from [39] to obtain the Heegaard diagram for $S^{1} \times \widehat{F}$. Start with a genus $g$ surface $A$ with two boundary components, $\alpha_{1}$ and $\beta_{1}$. Let $\xi_{2}, \ldots, \xi_{2 g+1}$ and $\eta_{2}, \ldots, \eta_{2 g+1}$ be two $2 g$-tuples of pairwise disjoint arcs in $A$ such that $\xi_{i}$ meets $\eta_{i}$ at a single point of transverse intersection, denoted $c_{i}$, and $\xi_{i} \cap \eta_{j}=\varnothing$ 


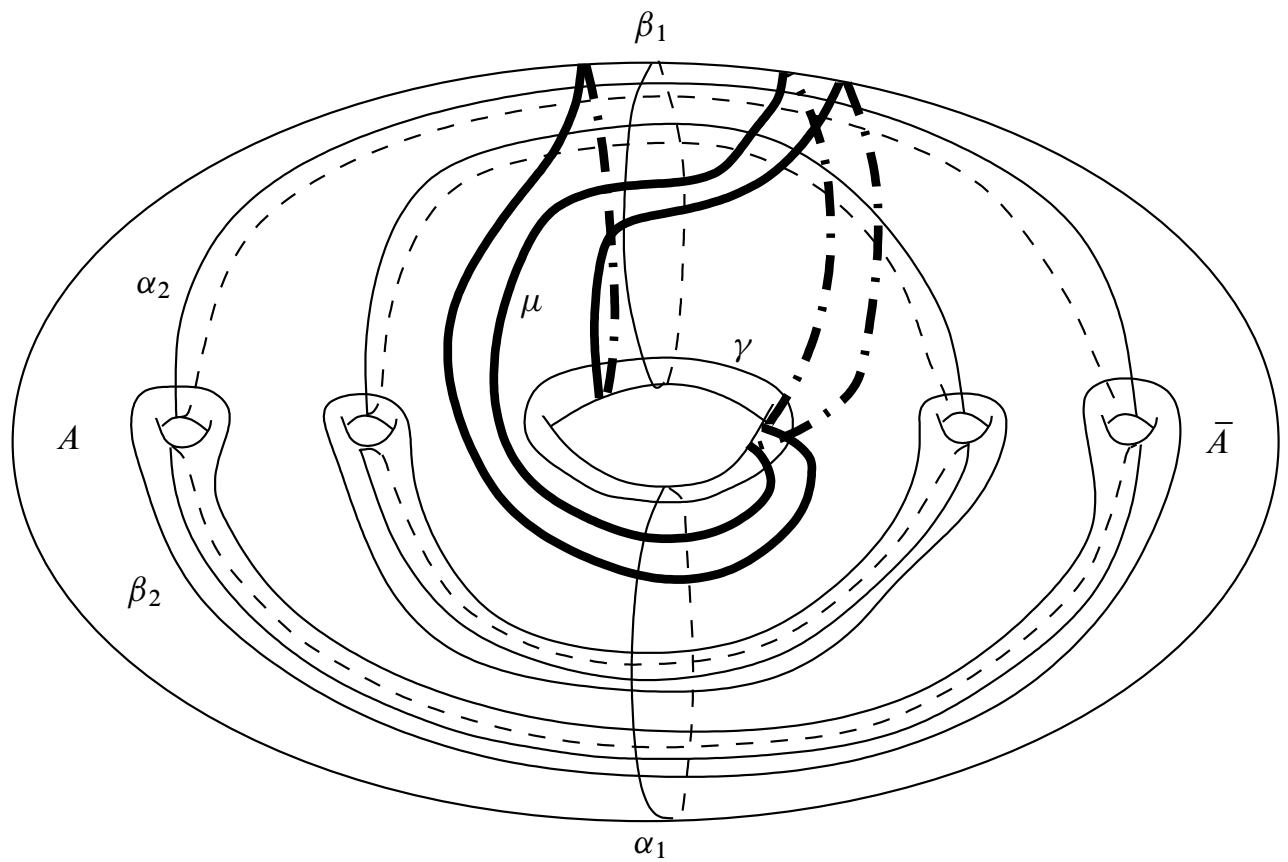

Figure 5: Constructing a Heegaard diagram from rational open book. The figure shows the Heegaard diagram arising form the open book with trivial monodromy. (To avoid overloading the picture, we have not drawn some of the $\alpha$-curves here. The missing curves lie in the back of the surface, in the top part of the diagram.) For a general open book, the $\beta$-curves in the $\bar{A}$ region will be affected by the action of the open book monodromy (this is not shown in the figure).

for $i \neq j$. A Heegaard diagram $\left(\Sigma,\left\{\alpha_{1}, \alpha_{2}, \ldots, \alpha_{2 g+1}\right\},\left\{\beta_{1}, \beta_{2}, \ldots, \beta_{2 g+1}\right\}\right)$ can then be obtained by doubling $A$ along its boundary; that is, we consider the surface $\bar{A}$ obtained by reflecting $A$ across its boundary, and glue $A$ and $\bar{A}$ together to form a closed surface $\Sigma$. This gluing produces closed curves $\alpha_{i}$, resp. $\beta_{i}, i=2, \ldots, 2 g+1$ by gluing $\xi_{i}$ to its copy $\bar{\xi}_{i}$, resp. $\eta_{i}$ to its copy $\bar{\eta}_{i}$. The result is a Heegaard diagram for $S^{1} \times \widehat{F}$. Moreover, removing $\beta_{1}$ results in a Heegaard diagram for the complement of $S^{1} \times\{\mathrm{pt}\} \subset S^{1} \times \widehat{F}$. This manifold is homeomorphic to the complement of the knot $B \subset \#^{2 g} S^{1} \times S^{2}$, where $B$ is the binding for the open book with trivial monodromy. The meridian of $B$ is represented by the curve $\gamma=\delta \cup \bar{\delta} \subset \Sigma$, formed by doubling an arc $\delta \subset A$ connecting $\beta_{1}$ and $\alpha_{1}$. These diagrams will be admissible after additional isotopies (finger moves) of the attaching circles [39]. 
To obtain a Heegaard diagram for $Y_{0}$, we must change the monodromy of the fibration. The monodromy map for $Y_{0}$ is the extension to $\widehat{F}$ of an automorphism $\phi: F \rightarrow F$. Thinking of $F$ as the complement of $\bar{\delta}$ in $\bar{A}$, we extend it by the identity to get an automorphism $\Phi: \Sigma \rightarrow \Sigma$. The diagram $\left(\Sigma, \boldsymbol{\alpha}, \boldsymbol{\beta}=\left\{\beta_{1}, \Phi\left(\beta_{2}\right), \ldots, \Phi\left(\beta_{2 g+1}\right)\right\}\right)$ represents $Y_{0}$, and $\left(\Sigma, \alpha,\left\{\Phi\left(\beta_{2}\right), \ldots, \Phi\left(\beta_{2 g+1}\right)\right\}\right)$ represents the complement of a fibered knot $\widetilde{K}$. With finger moves, these diagrams can be made weakly admissible for all $\mathrm{Spin}^{c}$ structures, as above.

Since $Y$ is a Dehn filling of the complement of $\widetilde{K} \subset Y_{0}$, we can obtain a Heegaard diagram for $Y$ by replacing $\beta_{1}$ by the meridian $\mu$ of $K \subset Y$. If $Y$ is obtained by a $p / q$-surgery on $\tilde{K}$ (with respect to the longitude given by $\gamma$ ), then $\mu$ can be represented by a curve on $\Sigma$ homologous to $p \beta_{1}+q \gamma$. A longitude for the knot $K$ is now given by a curve $\lambda$ on $\Sigma$ that intersects $\mu$ transversely at a single point, and is disjoint from the curves $\beta_{2}, \ldots, \beta_{2 g+1}$. Such a longitude is homologous to $b \beta_{1}+a \gamma$, for $a, b$ satisfying $p a-q b=1$. We may assume that, like $\mu$, the curve $\lambda$ is supported in a small neighborhood of $\beta_{1} \cup \gamma$.

The resulting Heegaard diagram is shown in Figure 5, and Figure 6 provides a closer look at the region containing $\mu, \beta_{1}$ and $\gamma$.

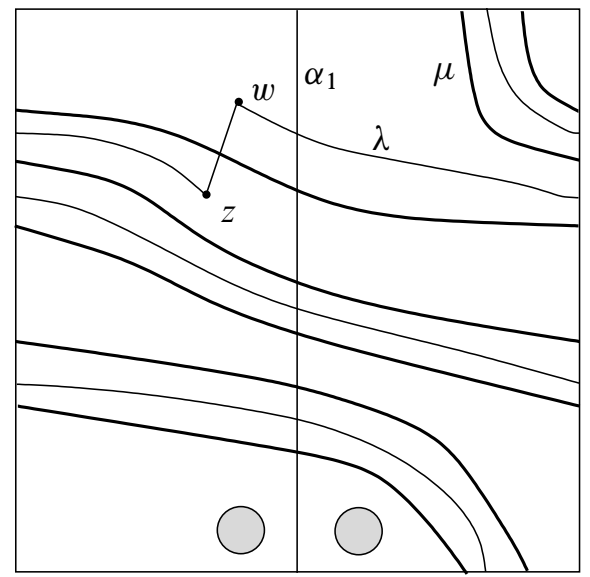

Figure 6: Zooming in on Figure 5: the meridian and the longitude of the binding of rational open book. The gray circles represent the remaining parts of Figure 5.

Observe that the Heegaard surface $\Sigma$ is cut by the attaching circles $\lambda, \mu$ and $\alpha_{1}$ into a large region lying in $A$, a large region lying in $\bar{A}$, a number of regions with boundary on $\lambda, \mu$ and $\alpha_{1}$, (see Figure 6), and a number of small regions in $\bar{A}$. 
Further observe that there is a 2-chain in $\Sigma$ whose boundary is $\alpha_{1}+\beta_{1}$. Since $\beta_{1}=a \mu-q \lambda$, we can find a relative periodic domain $\mathcal{P}$ whose homology class equals that of the fiber, with $\partial \mathcal{P}=\alpha_{1}+a \mu-q \lambda$. The multiplicities of $\mathcal{P}$ are 0 in the large region in $A$, and 1 in the large region in $\bar{A}$. The multiplicities in the regions in Figure 6 require a bit more work, but are also straightforward to compute. To find them, we start with 0 in the top left corner of Figure 6, and then move to the neighboring regions, changing the multiplicity by \pm 1 when we cross $\alpha_{1}$, by $\pm q$ when we cross $\lambda$ and by $\pm a$ when we cross $\mu$. (The signs depend on the direction in which the curves are traversed. In Figure 6, if travel upwards, the multiplicity of $\mathcal{P}$ increases when we cross $\lambda$, and decreases when we cross $\mu$. When crossing $\alpha_{1}$, the multiplicity increases by 1 from left to right.)

Our goal now is to show that there is a unique generator $\mathbf{x}$ that minimizes $n_{\mathbf{x}}(\mathcal{P})$. To begin, note that any generator must use both $\alpha_{1}$ and $\mu$ exactly once. We first argue that any generator minimizing $n_{\mathbf{x}}(\mathcal{P})$ has the form $\mathbf{x}=\left(x, x_{2}, \ldots, x_{2 g+1}\right)$, where $x \in \alpha_{1} \cap \mu$. Assume, to the contrary, that there is a generator $\mathbf{x}^{\prime}$ with minimal $n_{\mathbf{x}^{\prime}}(\mathcal{P})$, and that the intersection point of $\mathbf{x}^{\prime}$ on $\mu$ occurs on an $\alpha$ curve that is not $\alpha_{1}$. Denote this intersection point by $x^{\prime} \in \alpha_{i} \cap \mu, i \neq 1$. The four regions surrounding $x^{\prime}$ have local multiplicities given by $m, m, m+a, m+a$. This is because the boundary of $\mathcal{P}$ contains $\mu$ with multiplicity $-a$ and does not contain $\alpha_{i}$. Then there is an oriented arc along $\mu$, denoted $\kappa$, which intersects $\alpha_{1}$ positively in a point $x \in \mu \cap \alpha_{1}$, and which does not intersect $\alpha_{1}$ in any other points along the way. We examine how the multiplicities on either side of $\kappa$ change as we traverse its path from $x^{\prime}$ to $x$. First observe that if we intersect another $\alpha$ curve along the way, then the multiplicities do not change. This is because $\alpha_{1}$ is the only $\alpha$ curve occurring in $\partial \mathcal{P}$. The only other possibility for an intersection point occurring on $\kappa$ between $x^{\prime}$ and $x$ is if $\kappa \cap \lambda \neq \varnothing$. In this case, however, we can use the fact that $|\{\lambda \cap \mu\}|=1$ to perform an isotopy of $\lambda$ supported in a neighborhood of $\kappa$ that moves the point $\kappa \cap \lambda$ past the end of $\kappa$ to the other side of $\alpha_{1}$. Thus we may assume that the multiplicities on either side of $\kappa$ are $m$ and $m+a$. Since $\kappa$ intersects $\alpha_{1}$ positively, it follows that the multiplicities in the regions around $x$ are $m, m+a, m-1, m+a-1$. We would like to trade $x$ for $x^{\prime}$ to lower the multiplicities, thus arriving at a contradiction.

To do this, observe that the intersection point of $\mathbf{x}^{\prime}$ on $\alpha_{1}$ must

(1) be of the form $y^{\prime} \in \beta_{i} \cap \alpha_{1}$, and

(2) have $\mathcal{P}$ multiplicities $0,0,1,1$.

The reason for (2) is because $y^{\prime}$ occurs away from the regions surrounding $\mu \cup \lambda$, and $\partial \mathcal{P}$ contains $\alpha_{1}$ once. The reason for (1) is similar to Ozsváth and Szabó's argument: 
the curve $\beta_{i}$ must be used somewhere in $\mathbf{x}^{\prime}$. If it is not used for the intersection with $\alpha_{1}$, then it must be used in the region $\bar{A}$ where the multiplicities of $\mathcal{P}$ are 1 (since $\beta_{i}$ intersects only the two curves $\alpha_{i}$ and $\alpha_{1}$ inside $A$ ). This would result in the existence of a generator with smaller $n_{\mathbf{x}}(\mathcal{P})$. We can now make our trade to complete the first part of the argument. Indeed, consider the generator obtained from $\mathbf{x}^{\prime}$ by trading $x^{\prime} \in \alpha_{i} \cap \mu$ for $x \in \alpha_{1} \cap \mu$ and trading $y^{\prime} \in \beta_{i} \cap \alpha_{1}$ for the unique intersection point $c_{i} \in \alpha_{i} \cap \beta_{i}$ in $A$ (and keeping all other intersection points fixed). Calling this new generator $\mathbf{x}$, we have shown that $n_{\mathbf{x}}(\mathcal{P})=n_{\mathbf{x}^{\prime}}(\mathcal{P})-1$, contradicting the minimality of $\mathbf{x}^{\prime}$.

Thus a generator $\mathbf{x}=\left(x, x_{2}, \ldots, x_{2 g+1}\right)$ minimizing $n_{\mathbf{x}}(\mathcal{P})$ will have $x_{2}, \ldots, x_{2 g+1}$ contained in $A$, and $x \in \alpha_{1} \cap \mu$. Moreover, $\left\{x_{2}, \ldots, x_{2 g+1}\right\}=\left\{c_{2}, \ldots, c_{2 g+1}\right\}$ since these latter points are the unique intersection points in $A$ of the corresponding curves; in particular, the last $2 g$ coordinates of $\mathbf{x}$ are uniquely determined. The lowest value of $n_{\mathbf{x}}(\mathcal{P})$ will therefore be attained by those generators $\mathbf{x}=\left(x, c_{2}, \ldots, c_{2 g+1}\right)$ for which $n_{x}(\mathcal{P})$ is the lowest among all $x \in \alpha_{1} \cap \mu$.

To complete the proof of the proposition, we show that the values of $n_{x}(\mathcal{P})$ are mutually distinct for the various points $x \in \alpha_{1} \cap \mu$. If $n_{x}(\mathcal{P})=n_{x^{\prime}}(\mathcal{P})$, then the multiplicities at the four corners of $x$ and $x^{\prime}$ would be the same, since the multiplicities in the corners around $x$ and $x^{\prime}$ change in the same way when the curves $\alpha_{1}$ and $\mu$ are crossed. Consider, however, the shortest path from $x$ to $x^{\prime}$ along $\alpha_{1}$. If we cross the curve $\lambda$ a total of $r_{\lambda}$ times and the curve $\mu$ a total of $r_{\mu}$ times along this path, then we have $r_{\lambda} q-r_{\mu} a=0$. However, since $\lambda$ intersects $\alpha_{1}$ at $a$ points and $\mu$ intersects $\alpha_{1}$ at $q$ points, $0<r_{\lambda}<a$ and $0<r_{\mu}<q$. Thus $r_{\lambda} q-r_{\mu} a=0$ contradicts the fact that $\operatorname{gcd}(a, q)=1$. This shows that there is a unique point $c \in \alpha_{1} \cap \mu$ with smallest $n_{c}(\mathcal{P})$.

Remark 3.5 Theorem 1 and Proposition 3.4 provide two independent proofs of the fact that a rationally null-homologous fibered knot has knot Floer homology of rank 1 in the extremal Alexander grading. (This extends the analogous result for null-homologous knots, [39].) Yet another proof can be obtained by the sutured Floer homology of Juhász [26].

\section{The contact invariant of rational open books induced by surgery}

In this section we prove our non-vanishing theorem for the contact invariant of the contact structure induced by the rational open book that results from surgery on the binding of an honest open book. More precisely, recall that if we perform surgery on 
the binding of an honest open book, then the core of the surgery torus is a knot in the new manifold whose complement fibers over the circle (as it is homeomorphic to the complement of the original binding). Theorem 3 says that if the contact invariant associated to the original open book is non-zero, then the contact invariant of the induced rational open book is also non-zero, provided that the surgery parameter is sufficiently large.

Theorem 3 Let $K \subset Y$ be a fibered knot with genus $g$ fiber, and $\xi$ the contact structure induced by the associated open book. Let $K_{p / q} \subset Y_{p / q}$ be the rationally fibered knot arising as the core of the solid torus used to construct $p / q$ surgery on $K$, and $\xi_{p / q}$ the contact structure induced by the associated rational open book. Suppose $c(\xi) \in$ $\widehat{H F}(-Y)$ is non-zero. Then $c\left(\xi_{p / q}\right) \in \widehat{H F}\left(-Y_{p / q}\right)$ is non-zero for all $p / q \geq 2 g$.

We prove the theorem in steps, each step expanding the range of slopes for which the theorem holds. The first step is to show that the theorem holds for all sufficiently large integral slopes. This is accomplished by Theorem 4.4 below. The key tool in this step is an understanding of the relationship between the knot Floer homology of a knot $K \subset Y$ and the knot Floer homology of the core of the surgery torus $K_{n} \subset Y_{n}$. This relationship was studied in [19], following the ideas of [36]. We begin this section with a detailed discussion of these results, and prove a generalization (Theorem 4.2) which will serve as the cornerstone of our proof.

Our next step is to establish the theorem for all integral slopes $n \geq 2 g$. We accomplish this with Theorem 4.5, whose proof relies on a surgery exact sequence for the knot Floer homology of the core, together with an adjunction inequality.

Finally, we extend our results to all rational slopes $p / q \geq 2 g$. This argument is geometric, showing that the contact structures $\xi_{p / q}$ with rational slope can be obtained from those with integral slope by a sequence of Legendrian surgeries.

\subsection{The knot Floer homology of the core of the surgery torus}

We begin by stating a slightly rephrased version of [19, Theorem 4.1]. We use notation of [36].

Theorem 4.1 Let $K \subset Y$ be a null-homologous knot, and let $Y_{n}$ denote the manifold obtained by $n$-framed surgery on $K$. Then for all $n \gg 0$ sufficiently large, we have

$$
\widehat{C F}\left(Y_{n}, \mathfrak{s}_{m}\right) \simeq C\{\max (i, j-m)=0\},
$$

where $C\{\max (i, j-m)=0\}$ denotes the subquotient complex of $C F K^{\infty}(Y, K)$ whose $(i, j)$ filtration levels satisfy the stated constraint. 
Moreover, the core of the surgery torus induces a knot $K_{n} \subset Y_{n}$ and the filtration of $\widehat{C F}\left(Y_{n}, \mathfrak{s}_{m}\right)$ induced by (the reverse of) $K_{n}$ is filtered chain homotopy equivalent to the two-step filtration:

$$
0 \subset C\{i<0, j=m\} \subset C\{\max (i, j-m)=0\} .
$$

The first part of the theorem is simply [36, Theorem 4.4]. The second part, which deals with the filtration induced by $K_{n}$, was stated for $Y=S^{3}$ in the form above in [19, proof of Theorem 4.1, page 2304]. The proof carries through verbatim to general $Y$. We also note that the core of the surgery torus is isotopic to the meridian of $K$, viewed as a knot in $Y_{n}$. The original statement was phrased in these terms. We also remark that the formula above is, as indicated, for the knot $K_{n}$ with opposite the orientation it inherits from $K$. This was not discussed in [19], but the results there are independent of orientations. Here, too, knot orientation is not terribly important, but does affect the coorientation of contact structure so we choose to be careful throughout.

Since the contact invariant associated to $K_{n}$ is calculated using the bottom subcomplex in the knot Floer homology filtration of $K_{n}$, we need to understand what "bottom" means in the theorem above. Thinking of the filtration as a filtration by relative $\operatorname{Spin}^{c}$ structures on $Y_{n} \backslash v K_{n}$, the theorem above determines this difference in the case of relative $\operatorname{Spin}^{c}$ structures that project to the same absolute $\operatorname{Spin}^{c}$-structure on $Y_{n}$ under the natural filling map (1).

Thus we need to understand the difference between the relative $\operatorname{Spin}^{c}$ structures (or, if the reader prefers, the Alexander grading difference) associated to knot Floer homology groups for the varying $\mathfrak{s}_{m} \in \operatorname{Spin}^{c}\left(Y_{n}\right)$. Since the difference of two relative $\operatorname{Spin}^{c}$ structures lies in $H^{2}\left(Y_{n} \backslash v K_{n}, \partial\left(Y_{n} \backslash v K_{n}\right)\right) \cong H^{2}(Y) \oplus \mathbb{Z}$, we should make a few remarks about the algebraic topology of this situation. The first is to remind the reader that $Y_{n} \backslash v K_{n} \cong Y \backslash v K$, so the algebraic topology is, in a sense, identical. The key conceptual difference is that we have changed the natural framing on the boundary of this manifold. Thus, while $\mu_{K}$ generates the additional $\mathbb{Z}$ factor in $H_{1}(Y \backslash v K) \cong H_{1}(Y) \oplus \mathbb{Z}$, the meridian of $K_{n}$ does not generate the $\mathbb{Z}$ factor in $H_{1}\left(Y_{n} \backslash v K_{n}\right) \cong H_{1}(Y) \oplus \mathbb{Z}$. Indeed, $\left[\mu_{K_{n}}\right]=n \cdot \rho$ for a class generating this summand, and it is easy to see that $\rho=\left[K_{n}\right]$, the homology class of a push-off of $K_{n}$ into its complement.

Before stating the refined version of Theorem 4.1 we establish some notation. Let

$$
\begin{aligned}
S_{m} & =C\{i<0, j=m\} \\
Q_{m} & =C\{i=0, j \leq m\}
\end{aligned}
$$


be the sub- and quotient complexes in the filtration of $C\{\max (i, j-m)=0\}$ given by the theorem. The direct sum of all the knot Floer homology groups of $K_{n}$ (without the Alexander grading) is then given by

$$
\widehat{H F K}\left(Y_{n}, K_{n}\right)=\bigoplus_{m=-\lfloor n / 2\rfloor+1}^{\lfloor n / 2\rfloor} H_{*}\left(S_{m}\right) \oplus H_{*}\left(Q_{m}\right) \text {. }
$$

A complete description of the knot Floer homology of the core of the surgery is given by:

Theorem 4.2 Let $K \subset Y$ be a null-homologous knot and $K_{n} \subset Y_{n}$ be the (reverse of the) core of the surgery torus, viewed as a knot in the manifold obtained by $n$-framed surgery on $K$. Then for all $n \gg 0$ sufficiently large, the Alexander grading difference between the various knot Floer homology groups is given by

$$
\begin{aligned}
\mathrm{A}\left(S_{m}\right)-\mathrm{A}\left(Q_{m}\right) & =-n \\
\mathrm{~A}\left(S_{i}\right)-\mathrm{A}\left(S_{j}\right) & =-(i-j) \\
\mathrm{A}\left(Q_{i}\right)-\mathrm{A}\left(Q_{j}\right) & =-(i-j)
\end{aligned}
$$

for all $-\lfloor n / 2\rfloor+1 \leq m, i, j \leq\lfloor n / 2\rfloor$.

Remark 4.3 The filtration on $\widehat{C F}\left(Y_{n}\right)$ induced by $K_{n}$ is most easily understood graphically. For this we refer the reader to Figure 7.

Proof The proof is a straightforward extension of the proof of [19, Theorem 4.1], which, in turn, was an extension of the proof of [36, Theorem 4.4]. Both proofs were local, and involve an examination of the winding region in a Heegaard triple diagram representing the 2-handle cobordism from $Y_{n}$ to $Y$. See Figure 8 for a depiction of this region.

Given this Heegaard triple diagram, a chain map

$$
\widehat{C F}\left(Y_{n}, \mathfrak{s}_{m}\right) \longrightarrow C\{\max (i, j-m)=0\}
$$

is defined in [36] by counting pseudo-holomorphic triangles. The obvious small triangles present in the winding region (together with their extensions to $g$-tuples of small triangles in the rest of the triple diagram) induce a bijection of groups, provided that $n$ is large enough to ensure that all the intersection points for $\mathfrak{s}_{m}$ have $\alpha_{g}-$ component in the winding region. Moreover, these small triangles constitute the lowest order terms of the chain map with respect to the area filtration, and this latter fact shows that the chain map induces an isomorphism on homology. 


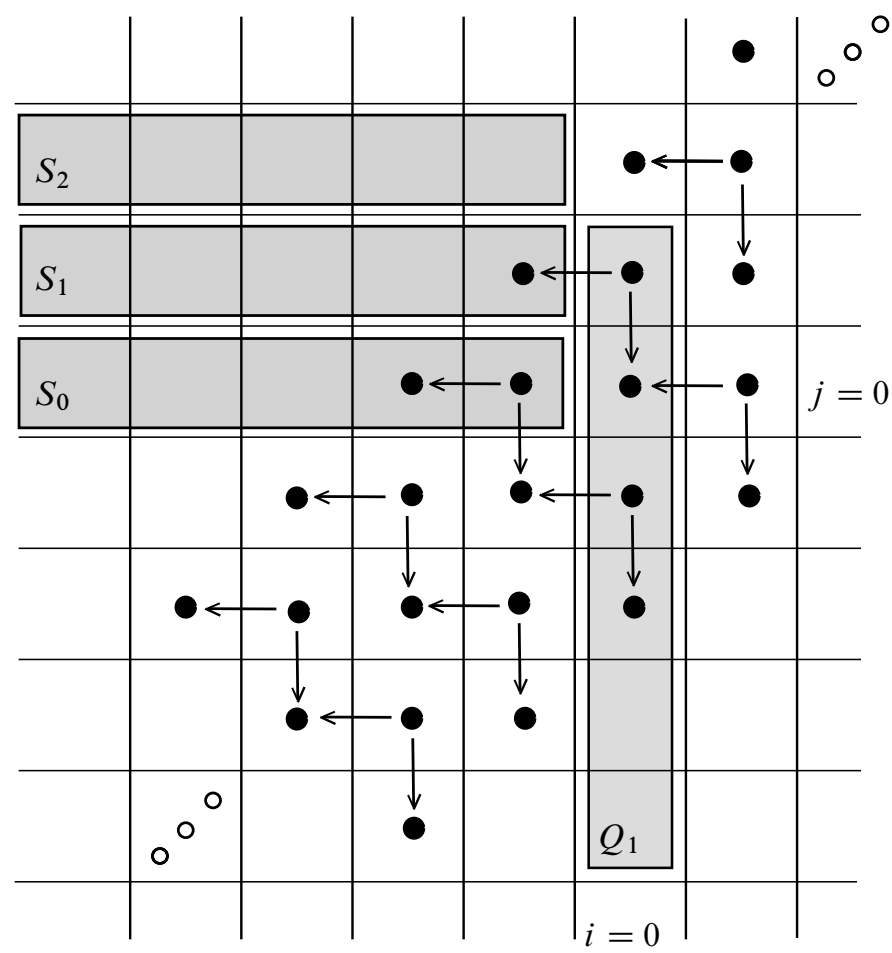

Figure 7: Shown is $C F K^{\infty}\left(S^{3}, K\right)$, for $K$ the $(2,5)$ torus knot. Dots equal $\mathbb{F}$, and arrows are non-trivial terms in the differential. The $\mathbb{Z} \oplus \mathbb{Z}$ filtration is given by the $(i, j)$ coordinates. The non-trivial knot Floer homology group for $K_{n} \subset S_{n}^{3}(K)$ with lowest Alexander grading is the homology of the subcomplex $S_{1}$ (while $S_{2}$ has lower Alexander grading, its homology is trivial). The homology of $S_{0}$ is the knot Floer homology group with Alexander grading 1 greater than that of $S_{1}$. The homology of $Q_{1}$ is the knot Floer homology group in Alexander grading $n$ greater than $S_{1}$.

To understand the filtration of $\widehat{C F}\left(Y_{n}, \mathfrak{s}_{m}\right)$ induced by $K_{n}$, we observe that the placement of a third basepoint $z^{\prime}$ on the Heegaard triple diagram has the property that $\left(\Sigma, \boldsymbol{\alpha}, \boldsymbol{\beta}, z^{\prime}, w\right)$ represents the reverse of $K_{n}$. The bijection induced by small triangles from the last paragraph is such that:

(1) If an intersection point for $\widehat{C F}\left(Y_{n}\right)$ has $\alpha_{g} \cap \beta_{g}$-component lying to the right of $x_{0} \in \alpha_{g} \cap \gamma_{g}$, then it is sent to a subcomplex $S_{m}$, with the distance to $x_{0}$ proportional to $-m$.

(2) If the $\alpha_{g} \cap \beta_{g}$-component is to the left of $x_{0} \in \alpha_{g} \cap \gamma_{g}$, the intersection point is sent to a quotient $Q_{m}$, with the distance to $x_{0}$ proportional to $-m$. 


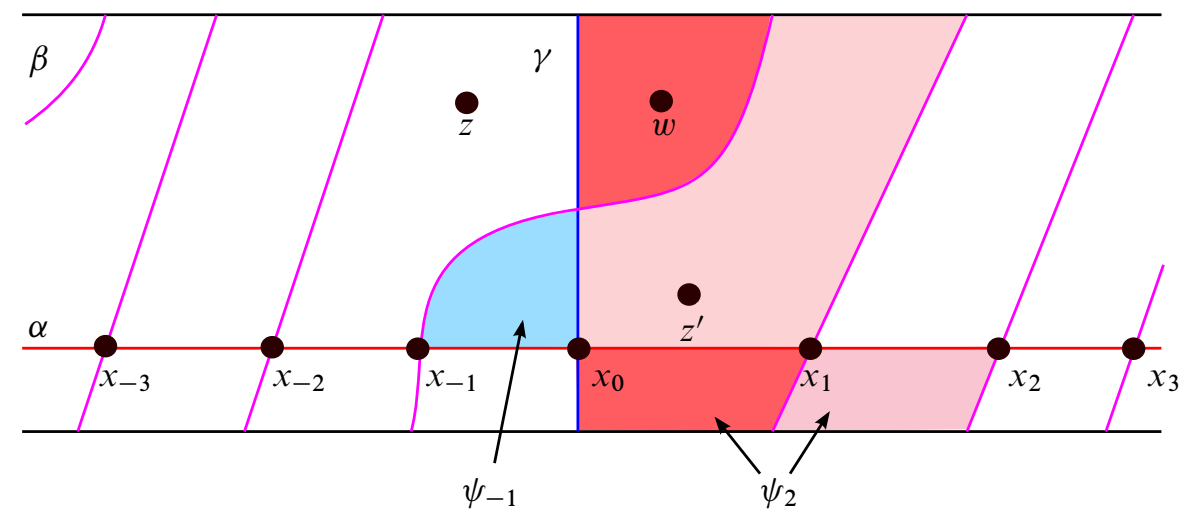

Figure 8: The "winding region" of the Heegaard triple diagram. A small triangle $\psi_{-1}$ connects $x_{-1}$ to $x_{0}$ and a small triangle $\psi_{2}$ connects $x_{2}$ to $x_{0}$.

Finally, any two intersection points $\mathbf{x}, \mathbf{y}$ representing $\mathfrak{s}_{m}$ can be connected by a Whitney disk $\phi$ that satisfies:

$$
n_{z^{\prime}}(\phi)-n_{w}(\phi)= \pm 1
$$

if the $\alpha_{g} \cap \beta_{g}$-components of $\mathbf{x}$ and $\mathbf{y}$ are on opposite sides of $x_{0}$, and

$$
n_{z^{\prime}}(\phi)-n_{w}(\phi)=0
$$

otherwise. Since

$$
\left(n_{z^{\prime}}(\phi)-n_{w}(\phi)\right) \cdot\left[\mu_{K_{n}}\right]=\epsilon(\mathbf{x}, \mathbf{y}) \in H_{1}\left(Y_{n} \backslash v K_{n}\right) \cong H_{1}(Y) \oplus \mathbb{Z}\left\langle\left[K_{n}\right]\right\rangle,
$$

and

$$
\left\langle\operatorname{PD}\left(\left[\mu_{K_{n}}\right]\right),[F, \partial F]\right\rangle=n\left[K_{n}\right] \cdot[F, \partial F]=n,
$$

this proves Theorem 4.1 (and the first part of the present generalization).

To complete the theorem, we must understand the filtration difference between the subcomplexes $S_{i}, S_{j}$ (respectively, the quotient complexes $Q_{i}, Q_{j}$ ) with $i \neq j$. By the transitivity of the filtration, it will suffice to understand the difference between $S_{i}$ and $S_{i+1}$. Consider a generator $\mathbf{x}=\left\{x_{-l}, \mathbf{s}\right\}$ lying in the subcomplex $S_{i}$, where $x_{-l} \in \alpha_{g} \cap \beta_{g}$ and $\mathbf{s}$ is the remaining $(g-1)$-tuple of intersection points. There is a corresponding generator $\mathbf{x}^{\prime}=\left\{x_{-l+1}, \mathbf{s}\right\}$, which lies in $S_{i+1}$, according to (1) above. These two generators can be connected by a curve that wraps once around the neck of the winding region; that is, $\epsilon\left(\mathbf{x}, \mathbf{x}^{\prime}\right)=\left[K_{n}\right]$, since this curve represents the generator of $H_{1}\left(Y_{n} \backslash K_{n}\right)$. Thus we have

$$
\mathrm{A}(\mathbf{x})-\mathrm{A}\left(\mathbf{x}^{\prime}\right)=\left\langle\mathrm{PD}\left(\left[K_{n}\right]\right),[F, \partial F]\right\rangle=1 .
$$


This proves the second line in the theorem. The third is given by a mirror argument on the left side of $x_{0}$.

\subsection{Non-vanishing for sufficiently large integral slopes}

With a firm understanding of the relationship between the knot Floer homology of $K \subset Y$ and $K_{n} \subset Y_{n}$, we can easily establish a non-vanishing theorem for sufficiently large integral surgeries.

Theorem 4.4 Suppose that the contact structure $\xi$, compatible with an open book $(Y, K)$, has $c(\xi) \neq 0$. For $n>0$, perform $n$-surgery on $K$, and consider the induced rational open book $\left(Y_{n}, K_{n}\right)$ and the compatible contact structure $\xi_{n}$. Then $c\left(\xi_{n}\right) \neq 0$ if $n$ is sufficiently large.

Proof Let $\xi$ be a contact structure compatible with an open book associated to a fibered knot $K \subset Y$, and let $\xi_{n}$ be the contact structure compatible with the rational open book associated to $K_{n} \subset Y_{n}$. By definition, the Ozsváth-Szabó contact element $c(\xi)$ is the image in $\widehat{H F}(-Y)$ of the generator of $H_{*}(\mathcal{F}(-Y, K$, bottom $)) \cong \mathbb{F}$, under the map induced by the inclusion:

$$
\iota: \mathcal{F}(-Y, K, \text { bottom }) \hookrightarrow \widehat{C F}(-Y) .
$$

By Theorem 1, this definition extends to rational open books. That is, the contact element $c\left(\xi_{n}\right)$ is equal to the image in $\widehat{H F}\left(-Y_{n}\right)$ of the generator of $\mathcal{F}\left(-Y_{n}, K_{n}\right.$, bottom) under the corresponding inclusion

$$
\iota^{n}: \mathcal{F}\left(-Y_{n}, K_{n}, \text { bottom }\right) \hookrightarrow \widehat{C F}\left(-Y_{n}\right) .
$$

To prove the theorem, we need only understand the relationship between the inclusion maps $\iota, \iota^{n}$, as governed by Theorem 4.2. Indeed, the theorem follows immediately from:

Claim Let $n$ be sufficiently large so that Theorem 4.2 holds. Then

$$
\iota_{*} \neq 0 \quad \Longleftrightarrow \quad \iota_{*}^{n} \neq 0 \text {. }
$$

To prove the claim, we first translate it into a statement about the topmost knot Floer homology group by a duality theorem. Throughout, we will use $K_{n}^{r}$ to denote the reverse of $K_{n}$. Consider the short exact sequence

$$
0 \longrightarrow \mathcal{F}(Y, K, \text { top }-1) \longrightarrow \widehat{C F}(Y) \longrightarrow \widehat{C F K}(Y, K, \text { top }) \rightarrow 0,
$$

and the associated connecting homomorphism

$$
\widehat{H F K}(Y, K, \text { top }) \stackrel{\delta_{*}}{\longrightarrow} H_{*}(\mathcal{F}(Y, K, \text { top }-1)) .
$$


A duality theorem [37, Proposition 2.5] states that the Floer homology of $-Y$ is the Floer cohomology of $Y$. The knot $K$ can be viewed in $-Y$, and there is a corresponding duality theorem for the filtrations [36, Proposition 3.7] (see also [20, Proposition 15] for the formulation we use here). In particular, the short exact sequence for $K \subset Y$ above is dual to the short exact sequence for $K \subset-Y$ :

$$
0 \longleftarrow \frac{\widehat{C F}(-Y)}{\mathcal{F}(-Y, K, \text { bottom })} \longleftarrow \widehat{C F}(-Y) \stackrel{\iota}{\longleftarrow} \mathcal{F}(-Y, K, \text { bottom }) \leftarrow 0,
$$

and so the connecting homomorphism $\partial_{*}$ for $-Y$ is dual to $\delta_{*}$. It follows that the kernel of $\delta_{*}$ is isomorphic to the cokernel of $\partial_{*}$ which, together with exactness, implies that:

$$
\iota_{*} \neq 0 \Longleftrightarrow \operatorname{ker} \delta_{*} \neq 0
$$

The duality theorem holds for rationally null-homologous knots, and thus the claim reduces to showing that

$$
\operatorname{ker} \delta_{*} \neq 0 \quad \Longleftrightarrow \quad \operatorname{ker} \delta_{*}^{n} \neq 0 .
$$

To do this, observe that Theorem 4.2 shows that $\widehat{H F K}\left(Y_{n}, K_{n}^{r}\right.$, top $) \cong H_{*}\left(Q_{-g}\right)$, the homology of the $-g^{\text {th }}$ quotient in the notation of that theorem, where $g=g(K)$ is the minimal genus of any embedded surface in the same homology class as $F$ (to see this, observe that $\mathcal{F}\left(Q_{i}\right)>\mathcal{F}\left(S_{j}\right)$ for all $i, j$, and that $H\left(Q_{i}\right)=0$ for all $i<-g(K)$, by the adjunction inequality [36, Theorem 5.1]). The map

$$
\delta_{*}^{n}: \widehat{H F K}_{*}\left(Y_{n}, K_{n}^{r}, \text { top }\right) \cong H_{*}\left(Q_{-g}\right) \longrightarrow H_{*-1}\left(\mathcal{F}\left(Y_{n}, K_{n}^{r} \text {, top }-1\right)\right)
$$

factors through the map induced by inclusion $S_{-g} \hookrightarrow \mathcal{F}\left(Y_{n}, K_{n}^{r}\right.$, top-1). Again, this follows from Theorem 4.2, as there are simply no generators in any other filtration levels that could be connected to those in $H_{*}\left(Q_{-g}\right)$ by Whitney disks. Thus ker $\delta_{*}^{n} \neq 0$ if and only if

$$
H_{*}\left(Q_{-g}\right) \longrightarrow H_{*-1}\left(S_{-g}\right)
$$

has non-trivial kernel or, equivalently, if

$$
H_{*}(C\{i=0, j=-g\}) \longrightarrow H_{*-1}(C\{i<0, j=-g\})
$$

has non-trivial kernel. But this map is the same, as a relatively graded map, as the map

$$
H_{*}(C\{i=0, j=g\}) \longrightarrow H_{*-1}(C\{i=0, j<g\})
$$

for the reverse of $K$, since reversing the orientation of $K$ interchanges the roles of $i$ and $j$ in the filtration [36, Proposition 3.8]. This last map is $\delta_{*}$ for the reverse of $K$. Thus $K_{n}^{r}$ has non-trivial invariant if and only if $K^{r}$ has non-trivial invariant. Reversing orientations a final time completes the proof of Theorem 4.4. 


\subsection{Non-vanishing for integral slopes $n \geq 2 g$}

Theorem 4.5 Suppose that the contact structure $\xi$, compatible with an open book $(Y, K)$ of genus $g$, has $c(\xi) \neq 0$. Then for all $n \geq 2 g$ the contact structure $\xi_{n}$ compatible with the induced rational open book $\left(Y_{n}, K_{n}\right)$ has $c\left(\xi_{n}\right) \neq 0$.

Proof Perhaps the most aesthetically appealing proof would be to show that Theorem 4.2 holds for all $n \geq 2 g$, regardless of the knot. We will take the easier route, and content ourselves to prove what is necessary for our application.

The proof makes use of a surgery exact sequence, together with an adjunction inequality. Recall the integer surgeries long exact sequence for the Floer homology of closed manifolds, which differ by surgery along a null-homologous knot $K \subset Y$ [37, Theorem 9.19]:

$$
\cdots \rightarrow \bigoplus_{i=1}^{n} \widehat{H F}(Y) \rightarrow \widehat{H F}\left(Y_{0}\right) \rightarrow \widehat{H F}\left(Y_{n}\right) \rightarrow \cdots
$$

This sequence holds for any framing $n>0$. Moreover, the sequence decomposes as a direct sum of $n$ exact sequences corresponding to the $\mathbb{Z} / n \mathbb{Z}$ factor in $H^{2}\left(Y_{n}\right) \cong$ $H^{2}(Y) \oplus \mathbb{Z} / n \mathbb{Z}$,

$$
\cdots \rightarrow \widehat{H F}(Y) \longrightarrow \underset{\left\{\mathfrak{s} \mid\left\langle c_{1}(\mathfrak{s}),[\widehat{F}]\right\rangle=2 m \bmod 2 n\right\}}{\bigoplus} \widehat{H F}\left(Y_{0}, \mathfrak{s}\right) \longrightarrow \widehat{H F}\left(Y_{n}, m\right) \rightarrow \cdots
$$

where $\widehat{H F}\left(Y_{n}, m\right)$ denotes the direct sum of the Floer homology groups associated to $\operatorname{Spin}^{c}$ structures on $Y_{n}$ that extend over the negative definite 2-handle cobordism $W$ from $Y_{n}$ to $Y$ to $\mathfrak{t} \in \operatorname{Spin}^{c}(W)$ satisfying $\left\langle c_{1}(\mathfrak{t}),[\hat{F}]\right\rangle+n=2 m$. Note we have stated the splitting in a somewhat more concrete form than [37, Theorem 9.19], implicitly using [34, Section 7; particularly Lemma 7.10]. We also note that the exact sequence further decomposes along $\mathfrak{s} \in \operatorname{Spin}^{c}(Y)$, but we will not need this structure.

We use a generalization of this exact sequence to the case of knot Floer homology. Let $K \subset Y$ be a null-homologous knot, and let $\mu \subset Y$ denote its meridian. We can view $\mu$ as knot in each of the three 3-manifolds of the sequence above, and consider their knot Floer homologies. Note that $\mu \subset Y$ is an unknot, and $\mu \subset Y_{0}$ (resp. $\mu \subset Y_{n}$ ) is isotopic to the core of the surgery, $K_{0}$ (resp. $K_{n}$ ). We have an exact sequence relating the knot Floer homology groups of these three knots

$$
\cdots \rightarrow \bigoplus_{i=1}^{n} \widehat{H F}(Y) \rightarrow \widehat{H F K}\left(Y_{0}, K_{0}\right) \rightarrow \widehat{H F K}\left(Y_{n}, K_{n}\right) \rightarrow \cdots
$$


where the first term is simply the Floer homology of $Y$, as $\mu$ is unknotted in this manifold. While such an exact sequence has not, to our knowledge, appeared explicitly in the literature, it is implicit from Ozsváth and Szabó's proof and nearly explicit in Eftekhary [8]. In any event, the sequence is easily obtained by adding an additional basepoint in the handle region of the Heegaard quadruple diagram where the surgery curve is being varied (recall Figure 8). It is then straightforward to go through the now standard technique for proving the existence of surgery exact sequences (see, for instance [40, Proof of Theorem 4.5]), requiring that all differentials, chain maps, chain homotopies, etc. are defined by counting $J$-holomorphic Whitney polygons that avoid both basepoints. As with the case of the Floer homology of closed 3-manifolds, we have a splitting of this exact sequence into $n$ sequences according to the $\operatorname{Spin}^{c}$ structures on $Y_{n}$ :

$$
\cdots \rightarrow \widehat{H F}(Y) \rightarrow \bigoplus_{\left\{\mathfrak{s} \mid\left\langle c_{1}(\mathfrak{s}),[\widehat{F}]\right\rangle=2 m \bmod 2 n\right\}} \widehat{H F K}\left(Y_{0}, K_{0}, \mathfrak{s}\right) \rightarrow \widehat{H F K}\left(Y_{n}, K_{n}, m\right) \rightarrow \cdots
$$

In addition, we know that the maps in the exact sequence are defined by counting $J$ holomorphic Whitney triangles associated to a doubly pointed Heegaard triple diagram. In each case there is a 4-manifold naturally associated to the triple diagram, and the first map is a sum over the triangle maps associated to homotopy classes whose $\mathrm{Spin}^{c}$ structure extends over the cobordism to $\mathfrak{s} \in \operatorname{Spin}^{c}\left(Y_{0}\right)$ satisfying $\left\langle c_{1}(\mathfrak{s}),[\widehat{F}]\right\rangle=2 \mathrm{~m}$. In particular, the component of the map coming from a fixed homotopy class of triangles is independent of $n$. Note that while these chain maps are likely an invariant of the embedded cylinder in the cobordism coming from the trace of $\mu$, we are not using this. We only use that the Heegaard triple diagram defining the first map is independent of $n$.

Given these exact sequences, we now apply Theorem 4.2. This tells us that

$$
\widehat{H F K}\left(Y_{n}, K_{n}, m\right) \cong H_{*}\left(S_{m}\right) \oplus H_{*}\left(Q_{m}\right),
$$

for sufficiently large $n$. The exact sequence, however, tells us that this group is also the homology of the mapping cone of

$$
\sum_{\mathfrak{t}_{m}} \widehat{F}_{W_{\mathrm{t}_{m}}}: \widehat{H F}(Y) \longrightarrow \bigoplus_{\left\{\mathfrak{s} \mid\left\langle c_{1}(\mathfrak{s}),[\widehat{F}]\right\rangle=2 m \bmod 2 n\right\}} \widehat{H F K}\left(Y_{0}, K_{0}, \mathfrak{s}\right)
$$

where the sum is over all $\operatorname{Spin}^{c}$ structures on the 2-handle cobordism whose Chern class is congruent to $2 m$, modulo $2 n$, and $\widehat{F}_{W_{\mathrm{t} m}}$ is the map defined by counting $J$-holomorphic triangles representing these $\operatorname{Spin}^{c}$ structures whose domains avoid both basepoints. 
The groups $\widehat{H F K}\left(Y_{0}, K_{0}\right)$ were first studied by Eftekhary [7], who referred to them as the longitude Floer homology groups. He showed [7, Theorem 1.1] that they satisfy an adjunction inequality, stating that $\widehat{H F K}\left(Y_{0}, K_{0}, \mathfrak{s}\right)=0$, unless

$$
-2 g+2 \leq\left\langle c_{1}(\mathfrak{s}),[\hat{F}]\right\rangle \leq 2 g .
$$

Here $g$ denotes the minimal genus of any Seifert surface in the relative homology class of a fixed surface $F$, and $\widehat{F}$ denotes this latter surface capped off by the disk in the solid torus of the zero surgery. Note that we have only stated the adjunction inequality aspect of [7, Theorem 1.1], which in fact says that the bounds above are sharp. Note, too, that our inequality is asymmetric, due to the fact that we used the map $\mathfrak{s}_{w}(-): \mathbb{T}_{\alpha} \cap \mathbb{T}_{\beta} \rightarrow \operatorname{Spin}^{c}\left(Y_{0}\right)$ coming from the basepoint $w$, whereas [7] uses the average $\frac{1}{2}\left(c_{1}\left(\mathfrak{s}_{w}(-)\right)+c_{1}\left(\mathfrak{s}_{z}(-)\right)\right)$, obtaining a symmetric inequality. The important aspect of the inequality is that it implies there are at most $2 g$ distinct $\operatorname{Spin}^{c}$ structures on $Y_{0}$ for which the middle term in the exact sequence is non-trivial. It follows that for $n \geq 2 g$, the groups under consideration $\widehat{H F K}\left(Y_{n}, K_{n}, m\right)$, are isomorphic to the mapping cone of

$$
\widehat{F}_{W_{\mathrm{t} m}}: \widehat{H F}(Y) \longrightarrow \widehat{H F K}\left(Y_{0}, K_{0}, \mathfrak{s}_{m}\right),
$$

where $\mathfrak{t}_{m}, \mathfrak{s}_{m}$ are the $\operatorname{Spin}^{c}$ structures on the cobordism and zero surgery, respectively, whose Chern classes satisfy (12). Since these maps are independent of $n$, it follows that (11) holds for all $n \geq 2 g$. Note, however, that the groups above are the knot Floer homology groups associated to all relative $\operatorname{Spin}^{c}$ structures on $Y_{n} \backslash K_{n}$ that project to $\mathfrak{s}_{m} \in \operatorname{Spin}^{c}\left(Y_{n}\right)$, under (1). Since our description of the contact invariant is in terms of the differential on the spectral sequence that starts at these groups and converges to $\widehat{H F}\left(Y_{n}, m\right)$, we must show that the filtration of $\widehat{C F}\left(Y_{n}, m\right)$ induced by $K_{n}$ agrees with the description of Theorem 4.2. (Note that (11) states only that the associated graded homology groups agree. We need to understand the entire filtration, and not simply the $E^{1}$-term of the corresponding spectral sequence.) In the case at hand, however, identification of filtrations is immediate. We are interested in the inclusion of the bottom subcomplex of the knot Floer homology filtration into the Floer homology of $-Y_{n}$ when $K_{n}$ is rationally fibered; namely, we would like to know whether the map on homology induced by the inclusion map

$$
\iota^{n}: \mathcal{F}\left(-Y_{n}, K_{n}, \text { bottom }\right) \hookrightarrow \widehat{C F}\left(-Y_{n}, m\right)
$$

is non-trivial. As in the proof of Theorem 4.4, this is the same question (via duality) as whether the kernel of the connecting homomorphism

$$
\delta_{*}^{n}: \widehat{H F K}\left(Y_{n}, K_{n}, \text { top }\right) \longrightarrow H_{*}\left(\mathcal{F}\left(Y_{n}, K_{n}, \text { top }-1\right)\right)
$$


is non-trivial. Since the top group has rank one homology, this is determined by the homologies $\widehat{H F}\left(Y_{n}\right)$ and

$$
\widehat{H F K}\left(Y_{n}, K_{n}\right) \cong H_{*}\left(S_{\text {top }}\right) \oplus H_{*}\left(Q_{\text {top }}\right) \cong H_{*}\left(S_{\text {top }}\right) \oplus \mathbb{F} .
$$

Here we have suppressed the relevant $\operatorname{Spin}^{c}$-structures on $Y_{n}$ in

$$
\widehat{H F}\left(Y_{n}\right) \text { and } \widehat{H F K}\left(Y_{n}, K_{n}\right)
$$

to avoid a notational conflict: in both cases we sum over $\operatorname{Spin}^{c}$ structures on $Y_{n}$ that extend over the two-handle cobordism to structures satisfying $\left\langle c_{1}(\mathfrak{t}),[\widehat{F}]\right\rangle+n=2$ (top). Bearing this in mind, the adjunction argument given above shows that for $n \geq 2 g$, $\widehat{H F K}\left(Y_{n}, K_{n}\right)$ can have at most two Alexander gradings with non-trivial knot Floer homology, ie, the filtration has at most two steps, with the quotient complex having rank 1 homology. The differential on $\widehat{H F K}\left(Y_{n}, K_{n}\right)$ that computes $\widehat{H F}\left(Y_{n}\right)$ is then identified with $\delta_{*}^{n}$. The group $\widehat{H F}\left(Y_{n}\right)$, however, is independent of $n$ once $n \geq 2 g-1$, by [36, Remark 4.3], so $\delta_{*}^{n}$ is the same for all $n \geq 2 g$. This completes the proof.

Remark 4.6 The key ingredient in our proofs of Theorems 4.4 and 4.5 is the understanding of the filtered chain homotopy type of $\widehat{C F K}\left(Y_{n}(K), K_{n}\right)$. For surgeries on a knot in $S^{3}$ (or more generally, in an integer homology L-space), this filtered chain complex can be understood via bordered Floer homology (Lipshitz, Ozsváth and Thurston [29, Sections 10,11]); in fact, the techniques of [29] provide the answer for an arbitrary knot and arbitrary surgery coefficient. However, [29] doesn't provide the answer for knots in an arbitrary 3-manifold $Y$; in any case, we find that a simple direct argument works better for our purposes.

\subsection{From integer to rational surgeries}

We have established Theorem 3 for the case of integral surgery. The following lemma extends Theorem 3 to rational surgeries. The proof of this lemma was explained to us by John Etnyre and Jeremy Van Horn-Morris.

Lemma 4.7 Let $(Y, K)$ be an open book decomposition compatible with the contact structure $\xi$. If $p / q>n>0$, the contact manifold $\left(Y_{p / q}, \xi_{p / q}\right)$ can be obtained from $\left(Y_{n}, \xi_{n}\right)$ by Legendrian surgery on a link.

Since the contact invariant is natural with respect to Legendrian surgeries, we have:

Corollary 4.8 If $p / q>n>0$ and $c\left(\xi_{n}\right)$ does not vanish, then $c\left(\xi_{p / q}\right)$ is non-zero. 
Proof of Lemma 4.7 We will prove the lemma by doing Legendrian surgery in certain toric slices (thickened tori) $T^{2} \times I$ inside $\left(Y_{n}, \xi_{n}\right) .(I=[0,1]$ is a closed interval.) We will take $T^{2}$ to be the boundary of a tubular neighborhood of the binding. In the argument below, we will be considering tori that are either convex (ie, those that are transverse to some vector field whose flow preserves the contact structure) or pre-Lagrangian (ie, those with linear characteristic foliation). Tight contact structures on $T^{2} \times I$ with convex boundary with fixed dividing set were classified in [22]. We will be using both Honda's classification and techniques from his paper. We assume that the reader is somewhat familiar with the convex surface theory (see Giroux [14], Honda [22] and Kanda [28]).

Before proceeding, we fix some notation. Consider the standard (Stein fillable) contact structure $\xi_{\text {std }}=\operatorname{ker}(\sin (2 \pi z) d x+\cos (2 \pi z) d y)$ on the 3-torus $T^{3}=T^{2} \times S^{1}$, where $z$ is the coordinate on $S^{1}$. Inside $T^{3}$, consider a convex torus isotopic to $T_{x, y}^{2} \times\{0\}$. The dividing set on such a torus consists of two parallel curves; let $s$ denote their slope. (We write $s=y / x$ for the slope of the line through $(0,0)$ and $(x, y)$.) Note that any $s \in[-\infty,+\infty]$ can be realized as a slope of some convex $2-$ torus inside $\left(T^{3}, \xi_{\text {std }}\right)$. Given two slopes $s_{0}$ and $s_{1}$ with $s_{1}>s_{0}$, we can find the convex tori $T_{0}$ and $T_{1}$ with corresponding slopes. They cut out a slice $T^{2} \times I$ out of $T^{3}$, such that $T_{0}$ is a front side of this slice and $T_{1}$ is the back side (with respect to the orientation given by $t$ on $S^{1}$ ). (The precise parametrization of $I$ is unimportant to us, so we will write $I$ for any interval, and abuse notation sometimes by using the same letter for a half-interval of $I$.) The contact structure $\xi_{\text {std }}$ is of course tight on this $T^{2} \times I$ slice and linearly rotating, ie, the dividing curves on the convex tori between $T_{0}$ and $T_{1}$ rotate linearly from slope $s_{0}$ on $T$ to slope $s_{1}$ on $T_{1}$. (Note the direction of rotation: it goes through large negative slopes, vertical slope, and then through large positive slopes.) Let $\left(T^{2} \times I, s_{0}, s_{1}, \xi_{\text {std }}\right)$ denote the toric slice we cut out of the Stein fillable $T^{3}$; we will always mean $\xi_{\text {std }}$ when we talk about a linearly rotating contact structure on a toric slice. In fact, there are two linearly rotating contact structures on a given slice; the 2-plane field is the same for both contact structures, but the co-orientation (the sign of the relative Euler class, [22, Sections 4.2, 4.3]) is different. Below, we consider a toric slice whose co-orientation is the same as that induced by the open book on our manifold.

To begin, consider an honest open book $(Y, K)$ with the induced contact structure $\xi$. Remove a small neighborhood of $K$ with convex boundary. For the torus $T^{2}=$ $\partial(Y \backslash \nu K)$ (oriented as the boundary of $Y \backslash v K)$, fix the identification $T^{2}=\mathbb{R}^{2} / \mathbb{Z}^{2}$ so that the longitude corresponds to $(1,0)$, and the meridian to $(0,1)$. There are two parallel dividing curves on this torus; let $s_{0}$ denote their slope. Notice that since $K$ is 
a transverse knot, we can assume that $s_{0}=-n_{0}$ for some integer $n_{0}>0$. (The number $n_{0}$ gets larger if we choose a smaller neighborhood of $K$.)

We will perform $n$-surgery on $(Y, \xi)$ by adding "extra rotation" in the neighborhood of the binding. Consider a toric slice $T^{2} \times I$ with a tight, minimally twisting, positively co-oriented contact structure $\zeta$ whose dividing curves rotate linearly from slope $s_{0}$ on $T_{0}=T^{2} \times\{0\}$ through larger negative slopes, vertical slope, and then through large positive slopes to a slope $s_{1}<n$. Inside this $T^{2} \times[0,1]$, we can find a pre-Lagrangian torus with slope $s$ for any $s$ in $\left(s_{0}, s_{1}\right)$ (this is easy to see because the contact structure is linearly rotating). Note that since $s_{0}>s_{1}$ in our case, the interval $\left(s_{0}, s_{1}\right)$ passes through $\pm \infty$, ie, is to be interpreted as $s \in\left(s_{0},+\infty\right) \cup\{ \pm \infty\} \cup\left(-\infty, s_{1}\right)$. Taking $s=n$, we find a slice isomorphic to $T^{2} \times[0,1]$ such that $T_{0}=T^{2} \times\{0\}$ is convex with slope $s_{0}, T_{1}=T^{2} \times\{1\}$ is pre-Lagrangian with slope $s_{1}=n$, and the contact structure is tight, minimally twisting, and linearly rotating as before.

Giroux's Flexibility Theorem [14; 22, Section 3.1.4] allows one to glue together contact manifolds along convex boundaries, provided that the dividing curves on the boundary surfaces match. Thus, we can attach the $T^{2} \times I$ slice described above to $Y \backslash v K$ so that $T^{2}=\partial(Y \backslash \nu K)$ is glued to $T_{0}$, and the dividing curves match. (Note that we pick the co-orientation of $\zeta$ to match that of the open book.) Now, $T_{1}=T^{2} \times\{1\}$ becomes the boundary torus; recall that it is pre-Lagrangian and has a linear characteristic foliation given by curves of slope $n$. The fibration of $Y \backslash v K$ by the pages of the open book $(Y, K)$ extends into $T^{2} \times[0,1]$ (compatibly with the contact structure). Collapsing to a point each leaf of the foliation of $T_{1}$, we get the surgered manifold $Y_{n}$, equipped with a well-defined contact structure and an open book decomposition. The contact structure is isotopic to $\xi_{n}$ and compatible with the open book: this is clear away from the binding, and we know that a contact structure extends uniquely over the binding [1, Theorem 1.7].

We will now perform Legendrian surgeries inside the slice $T^{2} \times I \subset(Y \backslash \nu K) \cup_{T_{0}}$ $T^{2} \times[0,1]$ to change the slope on $T_{1}$ to $p / q$. Collapsing the image of $T_{1}$ in the resulting contact manifold to circle as above, we will get an open book compatible with $\left(Y_{p / q}, \xi_{p / q}\right)$, together with a sequence of Legendrian surgeries that begin with $\left(Y_{n}, \xi_{n}\right)$ and terminate at $\left(Y_{p / q}, \xi_{p / q}\right)$.

By [22, Section 3.4.3], contact structures on $T^{2} \times[0,1]$ can be conveniently described using the Farey tessellation of the unit disk. By [22, Lemma 4.12], the contact structures we are interested in decompose into "bypass layers" as dictated by the Farey tessellation and the boundary slopes. (See [22] for details on how the decomposition works.) Each bypass layer is isomorphic to a "basic slice", ie, to $\left(T^{2} \times I, 1,0, \xi_{\text {std }}\right)$. Each basic slice comes with a sign [22, Proposition 4.7]. Since we have fixed the co-orientation on the linearly rotating contact structures above, all the bypass layers have the same sign. 
The tessellation picture (Figure 9) will help keep track of the effect that Legendrian surgeries have on the slope of $T_{1}$. Our toric slice $T^{2} \times I$ corresponds to the arc of the unit circle sweeping clockwise from $-\frac{n_{0}}{1}$ to $\frac{n}{1}$; thus we will be focusing on the left side of the tessellation disk.

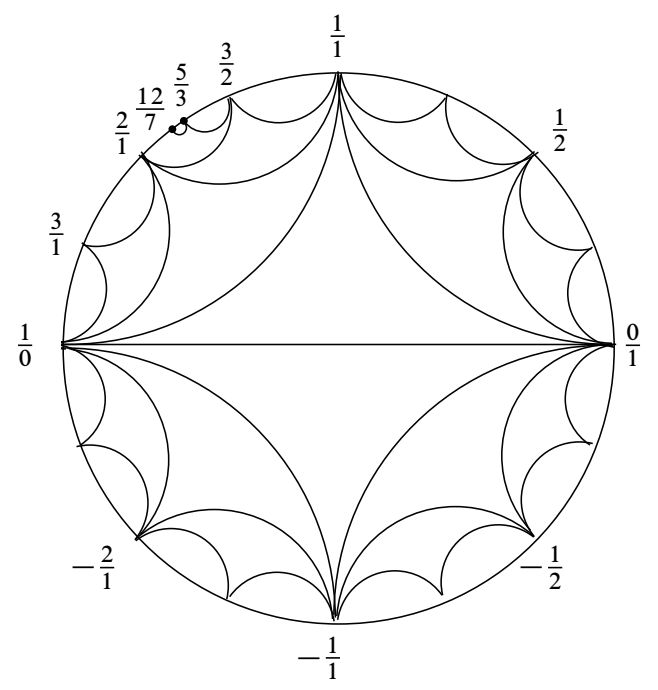

Figure 9: The Farey tessellation

Observe that if a toric slice with a linearly rotating contact structure has boundary slopes $s_{0}$ and $s_{1}$, then for any given rational slope $s$ between $s_{0}$ and $s_{1}$ there exists a pre-Lagrangian torus $T_{S}$ such that the leaves of its (linear) characteristic foliation have slope $s$. (This is easy to see because we consider a linearly rotating, ie, standard, contact structure.) Note that in our case, $s_{1}$ is always greater than $s_{0}$, so $s$ can vary in $\left(-\infty, s_{0}\right] \cup\left(s_{1}, \infty\right)$; this means that $s$ lies on the clockwise arc from $s_{0}$ to $s_{1}$.) We perform Legendrian surgery on a leaf of $T_{s}$. Here is the key observation.

Claim 4.9 Let $\left(T^{2} \times I, s_{0}, s_{1}, \xi_{\text {std }}\right)$ be a toric slice with a linearly rotating contact structure as considered above and a back slope $s_{1}=\frac{w}{t}$. Inside this slice, consider a pre-Lagrangian torus of slope $s=\frac{u}{v}>s_{1}$ such that there is a tessellation edge from $s$ to $s_{1}$, and perform a Legendrian surgery on a leaf of its foliation. Then after surgery, we obtain a toric slice $\left(T^{2} \times I, s_{0}, s^{\prime}, \xi_{\text {std }}\right)$, where the new back slope is $s^{\prime}=\frac{u+w}{v+t}$. (In other words, the new slope $s^{\prime}$ is the midpoint of the arc between $s$ and $s_{1}$, and can be reached from $s_{1}$ by hopping in the counterclockwise direction along a shorter edge.)

A similar claim can be found in Baldwin and Etnyre [4, Lemma 3.8]. It easy to see that the slope must transform as stated because the surgery can be interpreted as splitting along $T_{s}$ and regluing after a Dehn twist. The existence of an edge from $s$ to $s_{1}$ ensures 
that the curves corresponding to $(v, u)$ and $(t, w)$ intersect in $T^{2}$ homologically once. Thus, after the Dehn twist the slope on the boundary must be $s^{\prime}=(v+t, u+w)$. Having found the boundary slopes of the resulting contact structure on $T^{2} \times I$, we appeal to [22, Lemma 4.12] again to see that the contact structure decomposes into the bypass layers dictated by the Farey tessellation. The decomposition is clearly the same as that of $\left(T^{2} \times I, s_{0}, s^{\prime}, \xi_{\text {std }}\right)$. To complete the proof of the claim, we have to check that all bypass layers in the decomposition are negative (ie, have the same sign as the layers of the linearly rotating contact structure). The required analysis of signs can be found in [4, Lemma 3.8].

Since we work with open books obtained by collapsing the foliation on a pre-Lagrangian boundary of a toric slice, we need a slight variation of the claim above. Namely, we need to see that Legendrian surgery transforms the boundary slopes as stated in the claim for toric slices with pre-Lagrangian boundary (or for toric slices where the front boundary is convex and the back is pre-Lagrangian). However, this easily follows from the claim, because we can apply surgery to a smaller linearly rotating toric slice that is contained in our slice and has convex boundary. For this, recall that a basic slice (corresponding to an edge $[r, s]$ of the Farey tessellation) can be split into two halves corresponding to the two smaller edges connecting $r$ resp. $s$ to the midpoint of $[r, s]$. Each of the halves is isomorphic to the basic slice of the same sign as the original basic slice. Now, consider a toric slice whose front (convex) torus has slope $s_{0}$ and whose back (pre-Lagrangian) torus has slope $s_{1}$. Perform Legendrian surgery in a pre-Lagrangian torus of slope $s$ with a Farey edge from $s$ to $s_{1}$. Let $s^{\prime}$ be the midpoint of the arc $\left[s, s_{1}\right]$. We split the toric slice spanning the arc $\left[s, s_{1}\right]$ (with pre-Lagrangian boundary in the back) along the convex torus with slope $s^{\prime}$. The two resulting halves are "almost" basic slices (except that one of the boundary tori for each is pre-Lagrangian, not convex). Now, we can set aside the half corresponding to the $\operatorname{arc}\left[s^{\prime}, s_{1}\right]$ and apply the claim to surgery in the pre-Lagrangian torus of slope $s$ inside the toric slice with convex boundary spanning the arc $\left[s_{0}, s^{\prime}\right]$ in the Farey disk. The result of the surgery is $\left(T^{2} \times I, s_{0}, s^{\prime \prime}, \xi_{\text {std }}\right)$, where $s^{\prime \prime}$ is the midpoint of the arc between $s$ and $s^{\prime}$. Notice that all the basic slices we have here are of the same sign. Now, glue back the half-slice we set aside. Since this half-slice is isomorphic to the one spanning the arc $\left[s^{\prime \prime}, s^{\prime}\right]$, when we glue it to the half-slice spanning the arc $\left[s, s^{\prime \prime}\right]$, we get a "basic slice" $\left[s, s^{\prime}\right]$ (quotation marks are due to pre-Lagrangian boundary). It follows we recover the linearly rotating $T^{2} \times I$ with the front slope $s_{0}$ (for the convex boundary torus) and the back slope $s^{\prime}$ (for the pre-Lagrangian boundary torus).

Therefore, we have shown that Legendrian surgeries on leaves of the characteristic foliation on pre-Lagrangian tori relate our model contact structures to one another, changing the boundary slopes as predicted by the edges of the Farey tessellation. Now 
it remains to find the shortest sequence of edges connecting $\frac{n}{1}$ to $\frac{p}{q}$ in the tessellation picture, and perform the corresponding Legendrian surgeries. Suppose that $m$ is an integer such that $m+1>p / q>m$. If $m>n$, the sequence starts with hopping from $\frac{n}{1}$ to $\frac{m}{1}$ through integer slopes. Each of these hops corresponds to Legendrian surgery on a leaf in the pre-Lagrangian torus with slope $\frac{1}{0}$. Next, we continue along the edges from $m$ to $p / q$.

We illustrate this process by an example, describing a sequence of Legendrian surgeries that produces $\left(Y_{12 / 7}, \xi_{12 / 7}\right)$ from $\left(Y_{1 / 1}, \xi_{1 / 1}\right)$. Constructing the point $\frac{12}{7}$ in the tessellation disk, we get from $\frac{1}{1}$ to $\frac{12}{7}$ by moving along three edges: the edge from $\frac{1}{1}$ to $\frac{3}{2}$ (the midpoint of $\frac{1}{1}$ and $\frac{2}{1}$ ), then the edge from $\frac{3}{2}$ to $\frac{5}{3}$ (the midpoint of $\frac{3}{2}$ and $\frac{2}{1}$ ), then the edge from $\frac{5}{3}$ to $\frac{12}{7}$ (the midpoint of $\frac{5}{3}$ and $\frac{7}{4}$ ). These edges are shown on Figure 9. Therefore, $\left(Y_{12 / 7}, \xi_{12 / 7}\right)$ can be obtained from $\left(Y_{1 / 1}, \xi_{1 / 1}\right)$ by performing Legendrian surgery on the 3-component link consisting of two leaves of the characteristic foliation in the pre-Lagrangian torus with slope $\frac{2}{1}$, and a leaf of the foliation in the torus with slope $\frac{7}{4}$. The general case is treated similarly.

Remark 4.10 It is easy to see that under the hypotheses of Lemma 4.7, the manifold $Y_{p / q}$ carries a tight contact structure (with a non-vanishing invariant) for every $p / q>n$. Indeed, by the slam-dunk move [16], performing $p / q$-surgery on $K$ is equivalent to performing $n$-surgery on $K$, followed by $r$-surgery on the meridian of $K$, where $r=\frac{q}{q n-p}$. Since $r<0$, by [6] an $r$-surgery can be realized by a sequence of Legendrian surgeries, which results in a contact structure with non-vanishing contact invariant.

Lemma 4.7 establishes a stronger result: a specific contact structure $\xi_{p / q}$, arising from the given open book, has non-vanishing contact invariant $c\left(\xi_{p / q}\right)$.

\section{References}

[1] K L Baker, J B Etnyre, J Van Horn-Morris, Cabling, contact structures and mapping class monoids, J. Differential Geom. 90 (2012) 1-80 MR2891477

[2] J A Baldwin, Tight contact structures and genus one fibered knots, Algebr. Geom. Topol. 7 (2007) 701-735 MR2308961

[3] J A Baldwin, Heegaard Floer homology and genus one, one-boundary component open books, J. Topol. 1 (2008) 963-992 MR2461862

[4] J A Baldwin, J B Etnyre, Admissible transverse surgery does not preserve tightness arXiv: 1203.2993

[5] F Ding, H Geiges, A Legendrian surgery presentation of contact 3-manifolds, Math. Proc. Cambridge Philos. Soc. 136 (2004) 583-598 MR2055048

[6] F Ding, H Geiges, A I Stipsicz, Surgery diagrams for contact 3-manifolds, Turkish J. Math. 28 (2004) 41-74 MR2056760 
[7] E Eftekhary, Longitude Floer homology and the Whitehead double, Algebr. Geom. Topol. 5 (2005) 1389-1418 MR2171814

[8] E Eftekhary, Heegaard Floer homology and Morse surgery (2006) arXiv: math/0603171v2

[9] T Etgü, Tight contact structures on laminar free hyperbolic three-manifolds, Int. Math. Res. Not. 2012 (2012) 4775-4784 MR2989619

[10] J B Etnyre, K Honda, On the nonexistence of tight contact structures, Ann. of Math. 153 (2001) 749-766 MR1836287

[11] J B Etnyre, J Van Horn-Morris, Fibered transverse knots and the Bennequin bound, Int. Math. Res. Not. 2011 (2011) 1483-1509 MR2806512

[12] D T Gay, Symplectic 2-handles and transverse links, Trans. Amer. Math. Soc. 354 (2002) 1027-1047 MR1867371

[13] P Ghiggini, K Honda, J Van Horn-Morris, The vanishing of the contact invariant in the presence of torsion arXiv:0706.1602

[14] E Giroux, Convexité en topologie de contact, Comment. Math. Helv. 66 (1991) 637-677 MR1129802

[15] E Giroux, Structures de contact en dimension trois et bifurcations des feuilletages de surfaces, Invent. Math. 141 (2000) 615-689 MR1779622

[16] R E Gompf, A I Stipsicz, 4-manifolds and Kirby calculus, Graduate Studies in Mathematics 20, American Mathematical Society (1999) MR1707327

[17] J Greene, L-space surgeries, genus bounds, and the cabling conjecture arXiv: 1009. 1130

[18] M Hedden, On knot Floer homology and cabling, Algebr. Geom. Topol. 5 (2005) 1197-1222 MR2171808

[19] M Hedden, Knot Floer homology of Whitehead doubles, Geom. Topol. 11 (2007) 2277-2338 MR2372849

[20] M Hedden, An Ozsváth-Szabó Floer homology invariant of knots in a contact manifold, Adv. Math. 219 (2008) 89-117 MR2435421

[21] M Hedden, On Floer homology and the Berge conjecture on knots admitting lens space surgeries, Trans. Amer. Math. Soc. 363 (2011) 949-968 MR2728591

[22] K Honda, On the classification of tight contact structures, I, Geom. Topol. 4 (2000) 309-368 MR1786111

[23] K Honda, On the classification of tight contact structures, II, J. Differential Geom. 55 (2000) 83-143 MR1849027

[24] K Honda, W H Kazez, G Matić, Right-veering diffeomorphisms of compact surfaces with boundary, Invent. Math. 169 (2007) 427-449 MR2318562

[25] K Honda, W H Kazez, G Matić, On the contact class in Heegaard Floer homology, J. Differential Geom. 83 (2009) 289-311 MR2577470

[26] A Juhász, Holomorphic discs and sutured manifolds, Algebr. Geom. Topol. 6 (2006) 1429-1457 MR2253454 
[27] A Juhász, Floer homology and surface decompositions, Geom. Topol. 12 (2008) 299350 MR2390347

[28] Y Kanda, The classification of tight contact structures on the 3-torus, Comm. Anal. Geom. 5 (1997) 413-438 MR1487723

[29] R Lipshitz, P Ozsváth, D Thurston, Bordered Heegaard Floer homology: invariance and pairing arXiv:0810.0687

[30] P Lisca, A I Stipsicz, Ozsváth-Szabó invariants and tight contact three-manifolds, II, J. Differential Geom. 75 (2007) 109-141 MR2282726

[31] P Lisca, A I Stipsicz, Contact surgery and transverse invariants, J. Topol. 4 (2011) 817-834 MR2860344

[32] Y Ni, Knot Floer homology detects fibred knots, Invent. Math. 170 (2007) 577-608 MR2357503

[33] Y Ni, Link Floer homology detects the Thurston norm, Geom. Topol. 13 (2009) 29913019 MR2546619

[34] P Ozsváth, Z Szabó, Absolutely graded Floer homologies and intersection forms for four-manifolds with boundary, Adv. Math. 173 (2003) 179-261 MR1957829

[35] P Ozsváth, Z Szabó, Holomorphic disks and genus bounds, Geom. Topol. 8 (2004) 311-334 MR2023281

[36] P Ozsváth, Z Szabó, Holomorphic disks and knot invariants, Adv. Math. 186 (2004) 58-116 MR2065507

[37] P Ozsváth, Z Szabó, Holomorphic disks and three-manifold invariants: properties and applications, Ann. of Math. 159 (2004) 1159-1245 MR2113020

[38] P Ozsváth, Z Szabó, Holomorphic disks and topological invariants for closed threemanifolds, Ann. of Math. 159 (2004) 1027-1158 MR2113019

[39] P Ozsváth, Z Szabó, Heegaard Floer homology and contact structures, Duke Math. J. 129 (2005) 39-61 MR2153455

[40] P Ozsváth, Z Szabó, On the Heegaard Floer homology of branched double-covers, Adv. Math. 194 (2005) 1-33 MR2141852

[41] P Ozsváth, Z Szabó, Holomorphic disks, link invariants and the multi-variable Alexander polynomial, Algebr. Geom. Topol. 8 (2008) 615-692 MR2443092

[42] P Ozsváth, Z Szabó, Knot Floer homology and rational surgeries, Algebr. Geom. Topol. 11 (2011) 1-68 MR2764036

[43] J Rasmussen, Lens space surgeries and L-space homology spheres arXiv: 0710.2531

Department of Math, Michigan State University, D325 WH, East Lansing, MI 48823, USA Department of Mathematics, Stony Brook University, Stony Brook, NY 11794-3651, USA mhedden@math.msu.edu, olga@math.sunysb.edu http://www.math.msu.edu/ mhedden/Site/Home.html

Received: 8 May 2012 Revised: 13 October 2012 